\title{
THE SOLAR HEAVY ELEMENT ABUNDANCES. II. CONSTRAINTS FROM STELLAR ATMOSPHERES
}

\author{
M. H. Pinsonneault ${ }^{1}$ and Franck Delahaye $2,3,4$ \\ ${ }^{1}$ Department of Astronomy, The Ohio State University, Columbus, OH 43210, USA \\ 2 CEA, IRFU, Serv. Astrophys., F-91191 Gif-sur-Yvette, France \\ ${ }^{3}$ CEntre Lasers Intenses et Applications (CELIA), 351 cours de la libération, 33405 Talence Cedex, France \\ ${ }^{4}$ LUTH, (UMR 8102 associée au CNRS et à l'Université Paris 7), Observatoire de Paris, F-92195 Meudon, France \\ Received 2006 June 2; accepted 2009 September 3; published 2009 September 30
}

\begin{abstract}
Estimates of the bulk metal abundance of the Sun derived from the latest generation of model atmospheres are significantly lower than the earlier standard values. In Paper I, we demonstrated that helioseismic data combined with stellar interiors theory set strong bounds on the solar metal abundance. The seismically derived abundances are inconsistent with the low photospheric abundances if the quoted errors in the atmospheric models (of order 0.05 dex) are correct. In this paper, we undertake a critical analysis of the solar metallicity and its uncertainty from a model atmospheric perspective, focusing on CNO. We argue that the non-LTE (NLTE) corrections for abundances derived from atomic features are overestimated in the recent abundance studies, while systematic errors in the absolute abundances are underestimated. In general, abundances derived from molecular features are lower than those derived from atomic features for the three-dimensional hydro models, while a weaker trend in the opposite direction tends to hold for abundances derived from one-dimensional models. If we adopt the internal consistency between different indicators as a measure of goodness of fit, we obtain intermediate abundances $[\mathrm{C} / \mathrm{H}]=8.44 \pm 0.06,[\mathrm{~N} / \mathrm{H}]=7.96 \pm 0.10$ and $[\mathrm{O} / \mathrm{H}]=8.75 \pm 0.08$. The errors reflect the fact that both the high and low scales are internally consistent within the errors, and they are too large to conclude that there is a solar abundance problem. However, the center-to-limb continuum flux variations predicted in the simulations appear to be inconsistent with solar data based on recently published work. This would favor the traditional thermal structure and lead to high CNO abundances of $(8.52,7.96,8.80)$ close to the seismic scale. We argue that further empirical tests of NLTE corrections and the thermal structure are required for precise absolute abundances. The sensitivity of the simulations to spatial resolution and systematic errors in the underlying atmospheric physics should also be examined, and these effects may lead to an overestimate of the impact of convective overshooting on the thermal structure of the outer layers of the solar atmosphere. The uncertainties in the solar oxygen also imply that strong conclusions about the absence of solar beryllium depletion cannot be made.
\end{abstract}

Key words: convection - stars: abundances - Sun: abundances - Sun: evolution

Online-only material: color figure

\section{INTRODUCTION}

The uncertainty in the absolute chemical composition of stars is the limiting factor in our ability to do high-precision stellar astrophysics. Traditionally, we have had to rely on a small database of fundamental stellar parameters such as mass, distance, and radius. However, current and upcoming space missions promise a wealth of astrometric and photometric data. Large surveys undertaken primarily for other purposes (microlensing, planet searches, and cosmology) have discovered thousands of eclipsing binaries, yielding numerous precise mass estimates. The rapidly developing field of optical interferometry has also permitted a growing number of direct radius estimates. Asteroseismology is also growing in importance, and missions such as COROT promise a wealth of detailed information on the pulsational properties of solar-like stars.

Our stellar interiors models have become highly sophisticated and successful when compared with observational diagnostics. In particular, the resolution of the solar neutrino problem in favor of the solar model predictions and the agreement between theoretical predictions and helioseismic data are both encouraging signs. The combination of better observations and theory has opened the prospect of a new era of precision stellar astrophysics, which could have broad consequences for diverse subfields of astronomy.
Stellar atmospheric theory has traditionally employed a series of approximations when deriving abundances. Classical models assume an ad hoc turbulent velocity field adjusted to yield abundances independent of line strength. Convection is usually treated in an approximate fashion, with the mixing length theory. Horizontal temperature fluctuations (granulation) are not included. The models also typically assume that the molecular and atomic levels are described by local thermodynamic equilibrium (LTE), e.g., by the local temperature alone. The compilations of solar abundances used for theoretical solar models (Anders \& Grevesse 1989; Grevesse \& Noels 1993; Grevesse \& Sauval 1998) employed model atmospheres with approximations at the level described above. The mean thermal structure employed by these authors was semi-empirical (Holweger \& Mueller 1974, hereafter HM74). Other investigators have employed purely theoretical solar model atmospheres.

When these approximations are relaxed, different conclusions about the abundances are obtained. Departures from LTE are expected at a modest level for solar conditions, but can be significant for some elements and lines. They have been investigated by a number of authors (for example, Carlsson 1986; Stuerenburg \& Holweger 1990; Kiselman 1993; Shchukina \& Trujillo Bueno 2001; Wedemeyer 2001). Numerical simulations of convection have matured to the level where they can be used to predict velocity fields, temperature fluctuations, changes in the mean 
thermal structure of the upper atmosphere, and test the excitation of p-modes (Stein \& Nordlund 1998). Abundances derived from these simulations yield a very different pattern, which has been developed in a series of papers (Allende Prieto et al. 2001, 2002; Asplund 2000, 2004; Asplund et al. 2000a, 2000b, 2004, 2005c; Scott et al. 2006); papers by Lodders (2003) and Asplund et al. (2005a) summarize the revised abundance scale.

The net effect is in the sense of systematically lower metal abundances. The downward revisions for the heavier elements (e.g., $\mathrm{Fe}$ and $\mathrm{Si}$ ) are small, while the claimed reduction in the abundances of lighter species (especially CNO) is more dramatic. Two-dimensional convection simulations employing different treatments of granulation and non-LTE (NLTE) corrections (Holweger 2001; Steffen \& Holweger 2002) predict smaller abundance reductions. The central temperature predicted by interiors models is sensitive to the abundances of the heavier elements, but not the lighter ones. As a result, the new abundance scale does not disturb the agreement between interiors models and observational data for purposes such as the mass-luminosity relationship and solar neutrino fluxes.

However, the inferred solar sound speed profile, and the radii of interiors models, is sensitive to the bulk metallicity. Serious problems have emerged when comparing interiors models with the revised abundance scale. These discrepancies are evidence for problems in our understanding of stellar interiors, stellar atmospheres, or both. In Paper I (Delahaye \& Pinsonneault 2006), we investigated the errors in solar abundances predicted by the combination of stellar interiors models and helioseismic data. In this paper, we examine the uncertainties in the abundance predictions from stellar atmosphere theory. We begin with a brief summary of the results from Paper I, and follow with a discussion of the motivation and main results from the revised stellar atmospheric models. In Section 2, we perform a critical analysis of the precision of the solar $\mathrm{CNO}$ abundances and discuss the implications for Be. We demonstrate in that section that the errors in the abundances are larger than previously estimated, and that there is evidence that the "best" current solar CNO abundances are intermediate between the new and old scales, with errors permitting both. We discuss the implications of our finding and future tests in Section 3. In particular, we argue that inconsistencies between the solar thermal structure and that predicted by the simulations would favor a higher abundance scale closer to the seismic value and discuss uncertainties in the numerical convection simulations. Since the submission of the manuscript, several articles have been published treating different aspects of the uncertainties or new values for the revised abundances. We compare our results with these papers in the relevant sections. Our final recommended abundances are intermediate between the low and high scales discussed in this work and are similar to the recommendations in more recent publications, as discussed below.

\subsection{Constraints from Helioseismology}

Helioseismology provides two powerful constraints on the solar composition: diagnostics of the internal solar temperature gradient and diagnostics of the equation of state. Inversions of the observed solar pulsation frequencies yield accurate measures of the sound speed as a function of depth. In turn, the gradient in the sound speed can be directly tied to the temperature gradient. Since the temperature gradient is related to the opacity, and thus the composition, information on the solar abundances is encoded in the seismic data for the radiative interior. One can even obtain meaningful constraints on the solar age from the helium abundance profile deduced in the deep interior.

In addition to the vector information on the sound speed profile, there are also precise scalar quantities that can be extracted. The thermal structure at the base of the solar convection zone is nearly adiabatic, while the temperature gradient in the interior is radiative. As discussed in Paper I, Section 2.2, the resulting discontinuity in $\nabla$ generates a distinct signal that can be used to precisely localize the base of the solar convection zone $\left(R_{\mathrm{cz}}=0.7133 \pm 0.0005 R_{\mathrm{sun}}\right.$; Basu \& Antia 2004). Seismology also sets strict limits on convective overshooting $\left(<0.05 H_{P}\right.$; Christensen-Dalsgaard et al. 1995). The depth of the solar convection zone is sensitive to the light metal abundances in the Sun but insensitive to most of the other uncertainties in solar interiors models (see Paper I for a detailed error budget). Ionization also induces a depression in the adiabatic temperature gradient, and the absolute abundances of the species in question can be inferred from the magnitude of the perturbation in the surface convection zone. An extremely precise surface helium abundance can be deduced from this effect $\left(Y_{\text {surf }}=0.2483 \pm 0.0046\right.$, see Paper I, Section 2.3, for the sources used in this estimate). More recently, Antia \& Basu (2006) have demonstrated that the ionization signal of metals in the convection zone can be detected in the seismic data, leading to a bulk metallicity $Z=0.017 \pm 0.002$. Because the majority of the solar metals are in the form of $\mathrm{CNO}$, this is primarily a constraint on their abundance. In principle, one might be able to use this technique to solve for individual heavy element abundances by fitting the strength of distinct ionization stages. However, it is not yet clear that there is sufficient spatial resolution in the seismic data to permit such a detailed analysis.

In Paper I, we demonstrated that the combination of the surface convection zone depth and surface helium abundance constraints was a powerful diagnostic of the solar heavy element abundances. The surface helium abundance is tied to the initial solar helium abundance with a correction for gravitational settling. The initial helium is sensitive to the central opacity and the abundances of the heavier metals (especially iron). The convection zone depth is sensitive to the opacity at temperatures 2 million $\mathrm{K}$, where bound-free opacity from light metals (CNONe) is an important contributor. The most significant new finding in Paper I was that the combination of the two scalar constraints could be used to rule out some abundance combinations with high statistical significance. The detailed sound speed profile adds additional information; we found that models consistent with the scalar constraints could be constructed with low oxygen and very high neon, but such models exhibited substantial sound speed deviations relative to solar data in the deep interior. The key ingredient for constraining $\mathrm{Ne}$ is to examine models which reproduce the scalar constraints (surface helium abundance and convection zone depth) and then to examine the sound speed profile, rather than attempting to use the sound speed profile alone to constrain the model properties. The latter can produce degenerate solutions.

The inferred solar oxygen and iron abundances $([\mathrm{O} / \mathrm{H}]=$ $8.86 \pm 0.05,[\mathrm{Fe} / \mathrm{H}]=7.50 \pm 0.05)$ are consistent with the Grevesse \& Sauval (1998) absolute abundances, but strongly inconsistent with the new abundance scale within its quoted errors (Lodders 2003; Asplund et al. 2005a). Although there are potentially positive chemical evolution consequences for the revised abundance scale (Turck-Chièze et al. 2004), it is 
not easy to generate interiors models that are consistent with both seismology and the low abundance scale. The most commonly cited possible explanations on the interiors side (high neon, enhanced gravitational settling, and errors in the high temperature radiative opacities) are all strongly disfavored. As previously mentioned, solutions with high neon degrade agreement with the sound speed profile, and are also problematic from a solar coronal perspective (Schmelz et al. 2005); however, see also Drake \& Testa (2005) and Cunha et al. (2006) for an alternate perspective grounded in chemical evolution arguments. An increase in the degree of gravitational settling increases the convection zone depth but decreases surface helium, trading improved agreement with one diagnostic for worse agreement in another. Enhanced differential settling of metals with respect to helium is inconsistent with the underlying physics and would have to be extreme (Guzik et al. 2005). Three independent quantum mechanical calculations yield extremely similar Rosseland mean opacities at the temperatures of interest for the base of the solar convection zone (Iglesias \& Rogers 1996; Neuforge-Verheecke et al. 2001; Badnell et al. 2005). As discussed in Paper I, both the atomic physics and equation of state are relatively simple in this regime, and the concordance between different calculations is thus not a surprise.

One potential concern is the degree to which assumptions in the interiors models can impact their predictions. Fortunately, physical processes neglected in classical stellar models (such as rotational mixing and radiative acceleration) can be independently constrained by other data. In Paper I, we found that incorporating these effects would tend to induce higher rather than lower surface abundances. Mixing would reduce the degree of gravitational settling and would cause a shallower surface convection zone; radiative levitation would have a similar but smaller impact.

The scalar constraints are insensitive to the other theoretical ingredients in standard solar models (e.g., convection theory, surface boundary conditions, low temperature opacities, equation of state, and nuclear reaction rates). The considerations above indicate that it is extremely challenging to reconcile a low solar metal abundance with current stellar interiors models and seismic data. This does not imply that a metal-poor Sun is impossible, but it certainly motivates an investigation of the uncertainty in the atmospheric models used to derive the abundances.

\subsection{Model Atmosphere Ingredients}

The revised solar abundance estimates are derived from a variety of changes in the model atmospheres. Changes in oscillator strengths and equivalent widths of spectral lines contribute for some diagnostics, and as discussed below we largely concur with the revised values. The magnitude of NLTE corrections depends on the atomic model and the relative importance of photo-excitation and collisions on the level populations in the model atmosphere. The comprehensive re-examinations of the solar oxygen (Asplund et al. 2004, hereafter AGSAK04) and carbon (Asplund et al. 2005b, hereafter AGSAB05) adopted a particular set of assumptions for NLTE corrections, and we assess their uncertainties by comparison with limb darkening data and other published calculations. In Allende Prieto et al. (2001, 2002), the abundances derived from forbidden lines have been reduced by the application of blending corrections; AGSAK04 and AGSAB05 used the revised equivalent widths for $\mathrm{C}$ and $\mathrm{O}$ abundance studies. Both the uncertainty in these corrections and their central value are incorporated in our error analysis.
Three other coupled changes in the atmospheric models are the treatment of convective velocity fields ("macro/microturbulence"), horizontal temperature fluctuations (granulation), and the impact of convective overshooting on the mean thermal stratification. All of these features are derived from numerical convection simulations; a good discussion can be found in Stein $\&$ Nordlund (1998). Their combined impact can be deduced by the comparison of the results in the three-dimensional case in the published studies with the results from the semi-empirical one-dimensional HM74 thermal structure and the theoretical one-dimensional MARCS models. Since the three-dimensional model atmosphere is derived with physics (such as equation of state and opacities) similar to that in the MARCS code, the impact of the convection treatment can be indirectly inferred by comparing MARCS and three-dimensional abundances. A comparison of HM74 with MARCS and 3D is a measure of the impact of different choices of the thermal structure.

The numerical convection simulations predict line profiles in excellent agreement with the data for iron and silicon lines (Asplund et al. 2000a, 2000b). There are some trends with excitation potential that may be related to NLTE corrections (Shchukina \& Trujillo Bueno 2001) and issues with the initial generation of simulations when compared with line profiles in the outer solar photosphere (Scott et al. 2006). The concordance of the predicted amplitude of horizontal temperature fluctuations with the solar granulation pattern is encouraging (Stein \& Nordlund 1998; Asplund et al. 2000b). There is also an apparent conflict between the AGSAK04 simulations and the degree of convective penetration for the upper atmospheric layers (Ayres et al. 2006). However, the CO lines used in the latter analysis are formed very high in the atmosphere, which significantly complicates their analysis. Since these effects are all tied together in the abundance studies, we focus on the agreement between different diagnostics of abundance as a valuable test of the precision of the results obtained from different atmospheric models.

\section{THE SOLAR CNOBe ABUNDANCES}

Revised solar abundances have been derived for a number of species. In this paper, we focus on $\mathrm{CNO}$ for several reasons. First, these are the elements where the difference in abundance is largest, and they are also the cases where there is the largest variety of distinct abundance indicators. The difference between interiors and atmospheres based abundances for heavier species, such as $\mathrm{Fe}$ and $\mathrm{Si}$, is not statistically significant. Furthermore, details of the new abundance estimates and internally consistent comparison with prior work are published for only some of the heavier elements. The solar Be abundance is an important diagnostic of mixing, and the photospheric abundance is linked to the solar O (Balachandran \& Bell 1998). We therefore also briefly discuss the implications of our result for Be. We begin with a description of our overall approach, and then follow with individual sections on oxygen, carbon, and nitrogen. Our primary references for $\mathrm{O}, \mathrm{C}, \mathrm{N}$ are respectively AGSAK04, AGSAB05, and Asplund et al. (2005a). We include other studies for external comparisons, and discuss newer results based on other models and diagnostics when available.

\subsection{Overall Approach}

Our primary metric for the accuracy of the abundances derived from the different model atmospheres is the consistency of the estimates derived from distinct classes of indicators. One 
might justifiably apply a different standard, noting for example, the greater degree of sophistication in the input physics for the hydrodynamic simulations. However, it is not clear that the ingredients that induce the abundance changes (such as changes in the temperature gradient) are actually a necessary consequence of these improvements in the model, as the absolute errors in the first-principles theoretical models have not been quantified. We proceed in two steps. In the first, we compute relative abundances and errors within a given assumed model atmosphere for the atomic and molecular features. Since the abundances from atomic lines have smaller model-to-model differences (and thus smaller systematic errors), we adopt the atomic abundances for our base estimate. The difference between atomic and molecular abundances is then used to infer which of the different atomic scales should be adopted for the central value, and the uncertainty in the differential scales is used as a measure of the systematic error arising from the choice of model atmospheres.

For the atomic line diagnostics, we include random errors from the dispersion of results from single permitted lines about the adopted mean. For the forbidden lines, uncertainties in oscillator strengths and blending corrections become the dominant random error source. We also include systematic errors (NLTE corrections and zero-point shifts in the average oscillator strength) by comparing the study values with external constraints and other published calculations. This is important when comparing the atomic and molecular indicators, since NLTE corrections are usually included in the former but not the latter. As a result, changes in the degree of NLTE corrections have a direct impact on goodness of fit. When available, we adopt a weighted mean of the permitted and forbidden atomic indicators and the error in the mean when comparing with the molecular data.

For the molecular indicators, we include all measured lines of a given diagnostic and use the total dispersion (rather than the error in the mean) as a measure of goodness of fit. We adopt a weighted mean of the various indicators for an average molecular abundance. However, the error in the mean will understate the true uncertainty; it is frequently the case that the mean values for different molecular diagnostics differ by more than the dispersion within each indicator. We therefore compute the dispersion of each indicator about the adopted molecular mean and average these values to obtain an error in the molecular abundances. One could alternately compute the dispersion in the molecular abundances and treat this as a measure of the systematic uncertainty, adding it to the error in the mean in quadrature; this procedure yields somewhat smaller errors. Although the latter approach may be practical when there are numerous molecular probes available, we prefer the former method for situations like oxygen (where there are two values, and thus an unreliable estimate of the uncertainty in the mean).

We derive final abundance estimates for each species by comparing the mean abundances derived within each class of models for atomic and molecular indicators. In the case of oxygen, the three-dimensional and HM74 models exhibit comparable differences with opposite sign, while the MARCS models have an internally consistent intermediate abundance. We therefore adopt a mean of the different derived oxygen abundances and an uncertainty from the scatter. In the case of nitrogen, the HM74 abundances are preferred. The case of carbon depends on the origin of systematic differences in abundances inferred from atomic features. If the low zero point of AGSAB05 is adopted, the three-dimensional models are the
Table 1

Comparison Between the O Abundances Derived from Several Indicators

\begin{tabular}{lcccccc}
\hline \multicolumn{1}{c}{ Indicator } & Mean & Error & Mean & Error & Mean & Error \\
\hline & $3 \mathrm{D}$ & & HM04 & & M04 & \\
[O I $]$ & 8.68 & 0.01 & 8.76 & 0.02 & 8.72 & 0.01 \\
O I & 8.64 & 0.02 & 8.64 & 0.08 & 8.72 & 0.03 \\
OH $(v, r)$ & 8.61 & 0.03 & 8.87 & 0.03 & 8.74 & 0.03 \\
OH $(r, r)$ & 8.65 & 0.02 & 8.82 & 0.01 & 8.83 & 0.03 \\
Atomic & $\mathbf{8 . 6 7}$ & n/a & $\mathbf{8 . 7 5}$ & n/a & $\mathbf{8 . 7 2}$ & n/a \\
Molecular & $\mathbf{8 . 6 4}$ & n/a & $\mathbf{8 . 8 2}$ & n/a & $\mathbf{8 . 7 8}$ & n/a \\
Atom-Mol & $\mathbf{0 . 0 3}$ & n/a & $-\mathbf{0 . 0 7}$ & n/a & $\mathbf{0 . 0 6}$ & n/a \\
\hline & Rev3D & & RevHM & & RevMARCS & \\
[O I $]$ & 8.70 & 0.06 & 8.78 & 0.06 & 8.74 & 0.06 \\
O I $\left(S_{\mathrm{H}}=0\right)$ & 8.74 & 0.06 & 8.76 & 0.02 & 8.81 & 0.04 \\
O I $\left(S_{\mathrm{H}}=1\right)$ & 8.75 & 0.06 & 8.76 & 0.02 & 8.82 & 0.04 \\
OH $(v, r)$ & 8.61 & 0.03 & 8.87 & 0.03 & 8.74 & 0.03 \\
OH $(r, r)$ & 8.67 & 0.04 & 8.83 & 0.02 & 8.85 & 0.05 \\
Atomic $\left(S_{\mathrm{H}}=0\right)$ & $\mathbf{8 . 7 2}$ & $\mathbf{0 . 0 4}$ & $\mathbf{8 . 7 7}$ & $\mathbf{0 . 0 2}$ & $\mathbf{8 . 7 8}$ & $\mathbf{0 . 0 3}$ \\
Atomic $\left(S_{\mathrm{H}}=1\right)$ & $\mathbf{8 . 7 3}$ & $\mathbf{0 . 0 4}$ & $\mathbf{8 . 7 7}$ & $\mathbf{0 . 0 2}$ & $\mathbf{8 . 7 8}$ & $\mathbf{0 . 0 3}$ \\
Molecular & $\mathbf{8 . 6 3}$ & $\mathbf{0 . 0 3}$ & $\mathbf{8 . 8 4}$ & $\mathbf{0 . 0 4}$ & $\mathbf{8 . 7 6}$ & $\mathbf{0 . 0 6}$ \\
Atom-Mol $\left(S_{\mathrm{H}}=0\right)$ & $\mathbf{0 . 0 9}$ & $\mathbf{0 . 0 5}$ & $-\mathbf{0 . 0 7}$ & $\mathbf{0 . 0 4}$ & $\mathbf{0 . 0 2}$ & $\mathbf{0 . 0 7}$ \\
Atom-Mol $\left(S_{\mathrm{H}}=1\right)$ & $\mathbf{0 . 1 0}$ & $\mathbf{0 . 0 6}$ & $-\mathbf{0 . 0 7}$ & $\mathbf{0 . 0 3}$ & $\mathbf{0 . 0 2}$ & $\mathbf{0 . 0 7}$ \\
\hline
\end{tabular}

Note. The upper part of the tables recalls the value obtained by Asplund et al. (2005a) while the bottom part summarizes our reanalysis using NLTE correction with $S_{\mathrm{H}}=0$ and $S_{\mathrm{H}}=1$ scaled cases (see text for details).

favored solution. If the higher zero point of previous work is adopted, the situation is similar to that for oxygen.

\subsection{Oxygen Abundance Indicators}

AGSAK04 derived a low solar oxygen abundance $(8.66 \pm$ 0.05 ) from four distinct indicators: atomic lines (forbidden and permitted) and two different classes of infrared molecular lines $((\mathrm{v}, \mathrm{r})$ and $(\mathrm{r}, \mathrm{r}))$. All four indicators had formerly been used to obtain higher absolute oxygen abundance (8.83 to 8.90 ). Abundance estimates from all indicators are reduced in the theoretical atmospheres with substantial overshooting because lines become stronger for a fixed abundance in the presence of a steeper temperature gradient. Molecular abundances are reduced more than atomic ones because the cooler atmospheric structure of the three-dimensional hydro models changes the chemical equilibrium. Horizontal temperature variations also have a differential impact on the molecular abundances. AGSAK04 included other effects that reduced the abundances derived from atomic features without impacting the molecular indicators: a combination of changes in oscillator strengths, the inclusion of blending features, and their large inferred NLTE effects for the permitted lines.

In this section we discuss the uncertainties in each of these cases. We advocate a substantial decrease in the magnitude of the NLTE corrections for the $[\mathrm{O} / \mathrm{H}]$ derived from the permitted atomic oxygen lines, and an increased error in both the abundance derived from the forbidden line (from uncertainties in the oscillator strength) and the permitted lines (from uncertainties in the NLTE corrections). We also derive an increased error from the $[\mathrm{O} / \mathrm{H}]$ derived from the IR molecular lines from the internal scatter and trends with excitation potential, and argue that the correspondence between the trends in the MARCS and threedimensional models is evidence for errors in shared physical ingredients, such as the equation of state and opacity. Our basic results are summarized in Table 1. In Table 1, the upper part of the table repeats the mean abundances and errors for the four 
different indicators presented in AGSAK04 based on the line to line scatter. We present our revised estimates for the same three cases in the lower part. The last three rows in each sub-table give the mean discrepancy between atomic and molecular indicators. At the end of the section we synthesize this information to obtain our best estimate for the solar oxygen abundance. In each of the following subsections, the error estimates derived are internal ones. The differences between the results from the three classes of models are prima facia evidence that systematic errors are important. The systematic errors are discussed in the final subsection.

\subsubsection{Forbidden Oxygen Lines}

The forbidden oxygen line at $6300.3 \AA$ has traditionally yielded high oxygen abundances. Allende Prieto et al. (2001) argued that a nearby blended $\mathrm{Ni}$ line contributed significantly to the oxygen feature. They treated the continuum level, $\log \left(g f_{\mathrm{Ni}}\right)$, and the oxygen abundance as free parameters, but assumed that the line profiles as given from the simulations were exact. The inclusion of the Ni feature induced a direct reduction of $0.13 \mathrm{dex}$ in the inferred oxygen abundance. In addition, the usage of a three-dimensional model atmosphere structure led to a further reduction of 0.08 dex in the oxygen abundance to an estimated $[\mathrm{O} / \mathrm{H}]=8.69 \pm 0.05$. In AGSAK04 another forbidden line at $6363.7 \AA$ was considered as a second indicator. The authors reduced the equivalent width by $0.5 \mathrm{~m} \AA$ for an estimated contribution from a blended $\mathrm{CN}$ feature to obtain an oxygen equivalent width of $1.4 \mathrm{~m} \AA$, also implying a low abundance. We begin our analysis by noting that abundances derived from blended features are usually treated with caution. The most conservative procedure is to ignore the blending feature and treat the derived abundance as an upper limit. When this is done for the two forbidden lines, the maximum abundance obtained for 3D, HM, and MARCS are $(8.82,8.8),(8.9,8.88),(8.86,8.84)$, respectively. In the section that follows, we include the reduction in abundances from estimates of the blending contribution.

We adopt the AGSAK04 values for $[\mathrm{O} / \mathrm{H}]$ derived from the $6300.3 \AA$ line, subject to the caution on the strength of the $\mathrm{Ni}$ blending feature below. For the $6363.7 \AA$ line, Meléndez (2004) argued that the $(10,5) \mathrm{Q}_{2} 25.5 \mathrm{CN}$ line is unblended in the solar spectrum and has the same oscillator strength as the feature blended with the forbidden line. He derived a smaller correction for the blended $\mathrm{CN}$ line $(0.35 \mathrm{~m} \AA$ rather than the $0.5 \mathrm{~m} \AA$ value used in AGSAK04), which we adopt here. This leads to a modest 0.04 dex increase in the derived abundance from that line, which we also treat as an uncertainty in the $[\mathrm{O} / \mathrm{H}]$ derived from this feature from the uncertainty in the contribution of $\mathrm{CN}$ to the blended feature. The error analysis for a blended feature is more complex than the one that can be employed for an isolated line. The derived $[\mathrm{O} / \mathrm{H}]$ is sensitive to the continuum level, and an error component for this should be included; Allende Prieto et al. (2001) estimate this uncertainty at $0.02 \mathrm{dex}$, which we adopt for both lines. AGSAK04 adopted higher values for the oscillator strengths than those found in the NIST database, but their choice is well supported by the improved atomic physics (see Storey $\&$ Zeippen 2000). However, the errors in individual theoretical $\log (g f)$ values are higher than those assigned in Allende Prieto et al. (2001); we adopt 0.04 dex for individual lines. Allende Prieto et al. (2001) also estimated that uncertainties in the underlying equation of state induce a 0.02 dex error.

Especially for the $6300 \AA$ line, the results depend heavily on the detailed line profiles, particularly in cases where the individual components cannot be directly disentangled. Allende Prieto et al. (2001) estimated uncertainties of 0.02 dex from the central wavelength of the $\mathrm{Ni}$ feature and a 0.04 dex uncertainty from the central wavelength of the [O I] line. We treat these errors as representative of the uncertainties in the line profiles, and adopt them for both forbidden lines.

The treatment of the $\mathrm{Ni}$ line in the main forbidden line is more problematic. In the initial study, the oscillator strength was highly uncertain. Allende Prieto et al. (2001) treated log (gf) for $\mathrm{Ni}$ as a free parameter. The continuum level, $\log \left(g f_{\mathrm{Ni}}\right)$, and $[\mathrm{O} / \mathrm{H}]$ were treated as free parameters and the combination that produced the minimum $\chi^{2}$ was adopted. However, Johansson et al. (2003) have measured the oscillator strength of the $\mathrm{Ni}$ feature $(\log g f=-2.11)$, and the $\mathrm{Ni}$ abundance of the solar mixture is well constrained by knowledge of the solar $\mathrm{Si} / \mathrm{Fe}$ and the relative meteoritic abundances. With the new $g f$ value and $\log [\mathrm{Ni} / \mathrm{H}]=6.25$ the blending feature would be 0.2 dex stronger than the best $\chi^{2}$ value obtained in the 2001 paper. AGSAK04 did not report the inferred strength of the blending feature in their fit, but the similarity in the absolute abundance suggests that it would be comparable. We believe that it is no longer appropriate to treat this as a free parameter, and the same method should be used as is done for other blended features: namely, the strength of the $\mathrm{Ni}$ line should be held fixed (and varied within its uncertainty) while the free parameters are the oxygen abundance and continuum level.

The direct effect of increasing a blending contribution is usually to decrease the abundance, but the present case is more complicated. For the quoted values in the AGSAK04 fit, the Ni line contributes $25 \%$ of the total equivalent width of the line. An increase of 0.2 dex in the strength of the feature would imply a total $\mathrm{Ni}$ contribution of $40 \%$ of the blended equivalent width at a fixed continuum level; such a combination would be a poor fit to the line shape and would yield a reduction in the inferred oxygen of $0.11 \mathrm{dex}$. An increase in the continuum level would be required to restore the agreement with the line profile, which would in turn lead to an increased total equivalent width. The net impact on the derived oxygen is not obvious, and not necessarily in the negative direction. Reetz (1998) invoked $\log g f=-1.95$ for the $\mathrm{Ni}$ feature and obtained $[\mathrm{O} / \mathrm{H}]=$ 8.75 for the forbidden line when his oscillator strength for the forbidden line was adjusted to the same value as that employed by Allende Prieto et al.; however details of this work were not published and it is difficult to evaluate. In the absence of other information, we assign an additional error component of 0.04 dex for the strength of the Ni feature, slightly higher than the value advocated by Meléndez (2004). Adding the errors in quadrature, we obtain an uncertainty of 0.078 per line $(0.055$ in the average) and abundances derived from the forbidden lines systematically 0.02 dex higher than those found in AGSAK04.

After this paper was submitted, a number of investigators have studied this issue. Meléndez \& Asplund (2008) have advocated the usage of an additional forbidden oxygen feature at $5577 \mathrm{~A}$, and they obtain a relatively low value of $8.71 \pm$ 0.07 from this feature alone. Centeno \& Socas-Navarro (2008) used spectropolarimetry to disentangle the [O I] feature from the nickel blend, arriving at a high abundance of $8.86 \pm 0.07$. Scott et al. (2009) argue that a lower oxygen can still be obtained for this feature with a large downward revision in solar Ni to $6.17 \pm$ 0.05 . As noted above, this would cause significant helioseismic issues. Ayres (2008) argues that if the wavelength scale is held fixed (but the Ni abundance is not) then the best fit has higher $\mathrm{O}$ and lower Ni. Caffau et al. (2008) have an independent 
theoretical model from AGSAK04, and they performed an analysis close in spirit to ours. They obtained three-dimensional values for the CO5BOLD model of 8.68 and 8.78 for the 6300.3 A and 6363.7 A features, respectively. In both cases they note a significant dependence on the treatment of blended features; there are also measurable differences in the inferred abundance from the latter depending on the observational data set used. Their net inferred three-dimensional estimate, 8.73, is above our value of 8.68 for the three-dimensional Asplund et al. models; their HM estimate, 8.74 , is slightly below our value of 8.76. The former represents differences between threedimensional model atmosphere codes, while the latter reflects different analysis techniques. The overall situation appears to be subject to strong systematic effects, and we concur with the estimate of relatively high systematic blending errors by Caffau et al. (2008).

\subsubsection{Permitted Atomic Oxygen Lines}

The permitted atomic oxygen lines have relatively high oscillator strengths, but very high excitation potentials. The most commonly used features are the $\mathrm{O}$ I triplet at $7771.8,7774.2$, $7775.4 \AA$, with an excitation potential of $9.15 \mathrm{eV}$. AGSAK04 also considered three other atomic features $(6158.1 \AA$, $8446.7 \AA$, and $9266 \AA$ ). The primary reason for the low oxygen abundance inferred by AGSAK04 from the triplet is a large NLTE correction. Because these lines arise from such a high energy state, NLTE effects must be included. However, the quoted values of the NLTE corrections in the literature vary drastically. For the triplet, the average is -0.06 dex for Holweger (2001), -0.22 to -0.28 for AGSAK04, and -0.16 dex for Allende Prieto et al. (2004). These variations can be partially traced to different assumptions about the importance of collisional excitation (as opposed to photoionization), but differences at the $0.10 \mathrm{dex}$ level remain even for cases that make similar assumptions about hydrogen collisions.

AGSAK04 neglected hydrogen collisions in their estimate of the NLTE effects. They justified this by noting that for some well-studied lines, the classical Drawin (1968) formulism overestimates the collision rate. However, an inspection of their Figure 6 indicates that the neglect of collisional effects in their model yields changes as a function of limb darkening that differs from the observed solar values. This impression is confirmed by the more detailed study of Allende Prieto et al. (2004), who found that models including hydrogen collisions (their $S_{\mathrm{H}}=1$ case) were a better fit to the solar data. We therefore conclude that the NLTE corrections in AGSAK04 are overestimated, which has a significant effect on the concordance of the different oxygen indicators.

AGSAK04 applied larger downward reductions to the HM model than to the three-dimensional and MARCS models. Allende Prieto et al. (2004) indicate that similar corrections are obtained for Kurucz and three-dimensional models in their detailed study of the triplet as a function of limb darkening. There are therefore systematic differences in the differential NLTE corrections inferred by different investigators, which represents another systematic uncertainty. The magnitude of NLTE corrections is particularly important because the discordance between the oxygen derived for the one-dimensional models from atomic and molecular lines was used as a primary argument for the superiority of the three-dimensional models, and this discrepancy can be directly traced to the assignment of very large NLTE corrections to the HM model. By very similar logic, the internal dispersion in abundance for the atomic lines in the

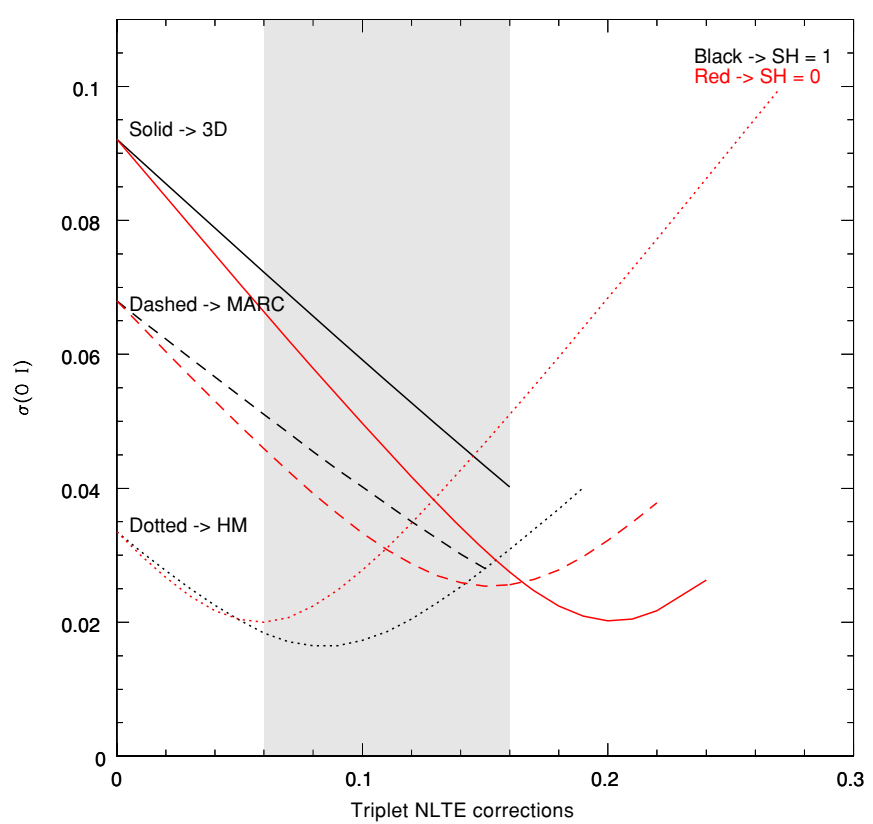

Figure 1. Internal scatter in oxygen inferred from permitted atomic lines as a function of the NLTE correction $\left(S_{\mathrm{H}}=0\right.$ case: $\mathrm{H}$ collisions are omitted and $S_{\mathrm{H}}=1$ when included) applied to the triplet. The solid line is the threedimensional model, the dotted line is the one-dimensional HM74 model, and the long-dashed line is the one-dimensional MARCS model. AGSAK04 adopted the values on the right side. The band denotes the limiting cases discussed in the text. The three-dimensional models are less internally consistent than the one-dimensional models for NLTE corrections in this range (see the text for details).

(A color version of this figure is available in the online journal.)

one-dimensional case arises from the assignment of large NLTE corrections to some of the lines; the internal agreement of the HM case is improved (and that of the three-dimensional and MARCS cases degraded) with smaller NLTE effects. We illustrate this point in Figure 1. We investigated two different normalizations: the published $S_{\mathrm{H}}=0$ case and an alternate $S_{\mathrm{H}}=1$ case (M. Asplund 2008, private communication.) In both cases, we scaled the NLTE corrections proportionally down, with the average triplet correction on the $x$-axis. We then computed the dispersion between indicators on the $y$-axis, and see that there is a strong dependence on the magnitude of the applied corrections. For the range of NLTE corrections that we consider reasonable (shaded band), the internal dispersion for the threedimensional models exceeds or is comparable to that of either one-dimensional model. This emphasizes the role of substantial NLTE corrections to the scientific conclusions of AGSAK04.

In order to quantify this effect, we normalized the NLTE corrections of AGSAK04 to an average for the triplet and explore the consequences of different methods for scaling the relative NLTE corrections for other lines. We adopt a benchmark triplet correction of 0.11 dex (the mean between Holweger 2001 and Allende Prieto et al. 2004) as our best case for reasons outlined below. The NLTE corrections for the other lines were scaled by the linear ratio of the average NLTE corrections for the triplet and the target values adopting two different absolute scalings: no $\mathrm{H}$ collisions $\left(S_{\mathrm{H}}=0\right)$ and full hydrogen collisions $\left(S_{\mathrm{H}}=1\right)$. We present revised abundance estimates for the permitted lines in AGSAK04 computed in this manner in Table 2. In Table 2, the first set of values contains the LTE results. The next set represents calculations where the NLTE corrections were normalized to obtain a mean triplet correction of 0.11 dex (our adopted mean). The final set of results includes the NLTE 
Table 2

Oxygen Abundance Derived from the O I Lines Using Different NLTE Corrections

\begin{tabular}{|c|c|c|c|c|c|}
\hline Indicator & $\begin{array}{c}\text { NLTE Triplet } \\
\text { Corrections }\end{array}$ & $\begin{array}{c}\text { Wavelength } \\
(\AA)\end{array}$ & $3 \mathrm{D}$ & HM04 & M04 \\
\hline O I & \multirow[t]{8}{*}{0.00 (LTE) } & 6158.1 & 8.65 & 8.82 & 8.80 \\
\hline $\mathrm{O}_{\mathrm{I}}$ & & 7771.9 & 8.91 & 8.89 & 8.95 \\
\hline $\mathrm{O}_{\mathrm{I}}$ & & 7774.2 & 8.89 & 8.87 & 8.94 \\
\hline $\mathrm{O}_{\mathrm{I}}$ & & 7775.4 & 8.86 & 8.86 & 8.91 \\
\hline $\mathrm{O}_{\mathrm{I}}$ & & 8446.7 & 8.80 & 8.83 & 8.88 \\
\hline $\mathrm{O}_{\mathrm{I}}$ & & 9266.01 & 8.73 & 8.79 & 8.77 \\
\hline Mean & & & 8.81 & 8.84 & 8.88 \\
\hline Dispersion & & & 0.10 & 0.04 & 0.07 \\
\hline \multicolumn{6}{|c|}{$S_{\mathrm{H}}=0\left(S_{\mathrm{H}}=1\right)$} \\
\hline $\mathrm{O}_{\mathrm{I}}$ & \multirow[t]{8}{*}{-0.11} & 6158.1 & $8.64(8.64)$ & $8.80(8.80)$ & $8.79(8.79)$ \\
\hline $\mathrm{O}_{\mathrm{I}}$ & & 7771.9 & $8.80(8.80)$ & $8.77(8.76)$ & $8.85(8.85)$ \\
\hline $\mathrm{O}_{\mathrm{I}}$ & & 7774.2 & $8.79(8.80)$ & $8.76(8.76)$ & $8.85(8.85)$ \\
\hline $\mathrm{O}_{\mathrm{I}}$ & & 7775.4 & 8.78 (8.79) & $8.76(8.76)$ & $8.83(8.84)$ \\
\hline $\mathrm{O}_{\mathrm{I}}$ & & 8446.7 & $8.72(8.74)$ & $8.73(8.74)$ & $8.79(8.82)$ \\
\hline $\mathrm{O}_{\mathrm{I}}$ & & 9266.01 & $8.70(8.71)$ & $8.74(8.75)$ & $8.74(8.75)$ \\
\hline Mean & & & $8.74(8.75)$ & $8.76(8.76)$ & $8.81(8.82)$ \\
\hline Dispersion & & & $0.06(0.06)$ & $0.02(0.02)$ & $0.04(0.04)$ \\
\hline \multicolumn{6}{|c|}{$S_{\mathrm{H}}=0\left(S_{\mathrm{H}}=1\right)$} \\
\hline $\mathrm{O}_{\mathrm{I}}$ & \multirow[t]{8}{*}{ Full NLTE } & 6158.1 & $8.62(8.64)$ & 8.77 (8.79) & 8.77 (8.79) \\
\hline $\mathrm{O}_{\mathrm{I}}$ & & 7771.9 & $8.64(8.71)$ & $8.60(8.67)$ & $8.71(8.78)$ \\
\hline $\mathrm{O}_{\mathrm{I}}$ & & 7774.2 & $8.65(8.73)$ & $8.60(8.68)$ & $8.71(8.79)$ \\
\hline $\mathrm{O}_{\mathrm{I}}$ & & 7775.4 & $8.66(8.73)$ & $8.62(8.69)$ & $8.71(8.78)$ \\
\hline $\mathrm{O}_{\mathrm{I}}$ & & 8446.7 & $8.60(8.70)$ & $8.58(8.68)$ & 8.67 (8.77) \\
\hline $\mathrm{O}_{\mathrm{I}}$ & & 9266.01 & 8.65 (8.69) & $8.68(8.72)$ & $8.69(8.73)$ \\
\hline Mean & & & $8.64(8.70)$ & $8.64(8.71)$ & $8.71(8.77)$ \\
\hline Dispersion & & & $0.02(0.03)$ & $0.07(0.05)$ & $0.03(0.02)$ \\
\hline
\end{tabular}

Notes. The top panel represent the LTE values followed by the results normalized to NLTE corrections of -0.11 dex for $S_{\mathrm{H}}=0$ and $S_{\mathrm{H}}=1$ in paranthesis respectively. The bottom panel recals the results from Asplund et al. (2004) and the new results from M. Asplund (2008, private communication) in parenthesis (see text for details).

corrections originally applied in AGSAK04 and those which would have been adopted if $\mathrm{H}$ collisions were included. This procedure yields NLTE $[\mathrm{O} / \mathrm{H}]$ abundances for three-dimensional models, one-dimensional HM74, and one-dimensional MARCS respectively of $8.74,8.76$, and 8.81 in the 0.11 dex case when the NLTE corrections with $S_{\mathrm{H}}=0$ are applied. In the case where $S_{\mathrm{H}}=1$ the NLTE abundances are 8.75, 8.76, and 8.82 for three-dimensional models, one-dimensional HM74, and onedimensional MARCS, respectively.

Since the NLTE corrections are significant for the triplet, the uncertainty in these corrections is a major ingredient in the error budget. Even the reduced NLTE corrections of Allende Prieto et al. (2004) for the triplet are substantially larger than the corrections used by Holweger (2001), who found an average NLTE correction of $-0.06 \mathrm{dex}, 0.10$ dex lower than the value reported by AGSAK04. Surprisingly, none of the authors involved commented on the origin of the difference. Holweger (2001) obtained average LTE and NLTE triplet abundances of 8.78 and 8.72, respectively; his NLTE abundance is close to that obtained for the two one-dimensional models in AGSAK04. From Allende Prieto et al. (2004), the case with no hydrogen collisions was ruled out at the $3 \sigma$ level, and LTE models were ruled out with high confidence. However, the authors did not consider whether even lower NLTE corrections than their $S_{\mathrm{H}}=1$ case would have provided improved fits to the data. In cases such as this, we see no justification for simply adopting one NLTE correction ( $0.16 \mathrm{dex})$ over another published value ( $0.06 \mathrm{dex})$, and adopt the average of the two $(0.11 \mathrm{dex})$. We note that our central values are close to what we would infer if we simply took the triplet alone as an oxygen abundance indicator and assigned a 0.16 dex NLTE correction.

We used the dispersion in the abundances derived from individual lines as a base random error. A change of 0.05 dex in the triplet NLTE correction yields an average change in $[\mathrm{O} / \mathrm{H}]$ of 0.035 dex, which we include as an additional systematic error. Finally, the $\log (g f)$ values from AGSAK04 are lower than previously published values by Biemont et al. (1991); we therefore add another 0.025 dex systematic error, following a similar error analysis by Meléndez (2004). The net effect is a total error estimate of $0.066(0.075 \mathrm{dex})$ for the threedimensional model (respectively for $S_{\mathrm{H}}=0$ and $S_{H}=1$ in parenthesis), 0.055 (0.048 dex) for the HM one-dimensional model and 0.055 ( $0.060 \mathrm{dex})$ for the one-dimensional MARCS model.

Since this paper was submitted there have been some noteworthy developments (see Barklem 2007; Socas-Navarro \& Norton 2007 for work on NLTE corrections). Caffau et al. (2008) note that the 6158.1 A feature is in a complex region of the spectrum, and that continuum placement may be an issue; blending is a problem for the 8446.7 A line, while a telluric feature contaminates the $9266.01 \mathrm{~A}$ feature. They advocate for an intermediate NLTE triplet correction, comparable in magnitude to that which we obtain for intensity measurements and larger for flux. Their oxygen abundance from permitted atomic lines would be 8.76 using the CO5BOLD code, compared with our revised threedimensional AGSAK04 measurement of 8.74-8.75. The value which they would have obtained for their HM model, 8.77, is close to our value of 8.76 . 

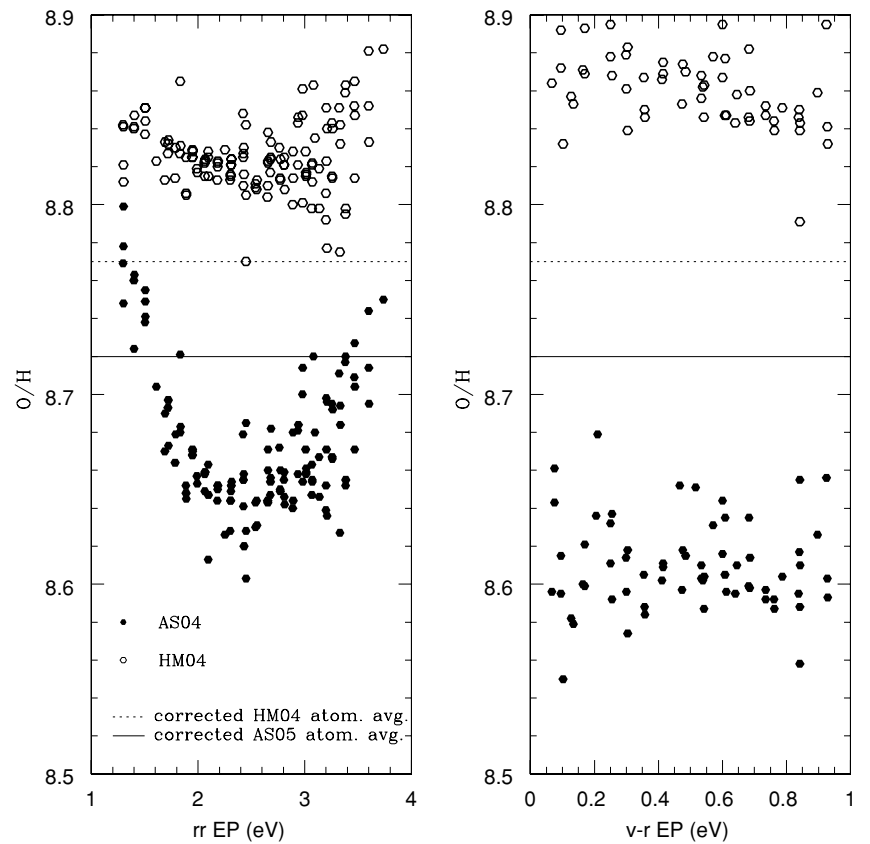

Figure 2. Oxygen abundances derived from infrared $\mathrm{OH}$ lines in the one-dimensional HM74 and three-dimensional AGSAK04 atmospheres are compared as a function of excitation potential. The left panel represents $(r, r)$ transitions, while the right panel represents (v,r) transitions. Results from the three-dimensional model atmospheres are solid circles; results from the HM74 semi-empirical model atmosphere are open circles. The solid and dashed lines indicate the mean abundances that would be derived from atomic features in the three-dimensional and HM74 models. Comparable discrepancies between molecular and atomic abundances are present for both classes of models, and the three-dimensional models exhibit striking trends with excitation potential not seen in the HM74 case.

\subsubsection{Infrared Molecular Lines}

The IR molecular oxygen lines have been considered a primary abundance indicator used in previous compilations of solar abundances (e.g., Grevesse \& Sauval 1998), and onedimensional model atmospheres yield relatively high absolute oxygen abundances. The absolute abundances are a strong function of the thermal structure of the model atmospheres, and the different thermal structure of the three-dimensional model of AGSAK04 yields much lower predicted abundances than the one-dimensional models. We note that Holweger (2001) discounted $\mathrm{CNO}$ abundances derived from molecular lines because of their high temperature sensitivity.

AGSAK04 considered two sets of $\mathrm{OH}$ lines: $(\mathrm{v}, \mathrm{r})$ and $(\mathrm{r}, \mathrm{r})$. The abundances predicted as a function of excitation potential from the three-dimensional hydro simulations are compared with those from the one-dimensional Holweger-Mueller and MARCS codes in Figures 2 and 3. On these figures we have also indicated the average atomic abundances for each class of models. We note the presence of striking trends with excitation potential in the three-dimensional and MARCS models for the $(r, r)$ lines. The correspondence between the MARCS and threedimensional trends indicates that the origin of these features is common to both models, which indicates that it is a feature of the base atmospheric treatment rather than being induced by the convection simulation.

In the context of one-dimensional models, trends such as those seen in the three-dimensional models would be interpreted as a problem with the thermal structure or the assumed microturbulence. AGSAK04 noted this trend, and claimed that it could be removed by invoking an outer atmosphere structure even cooler
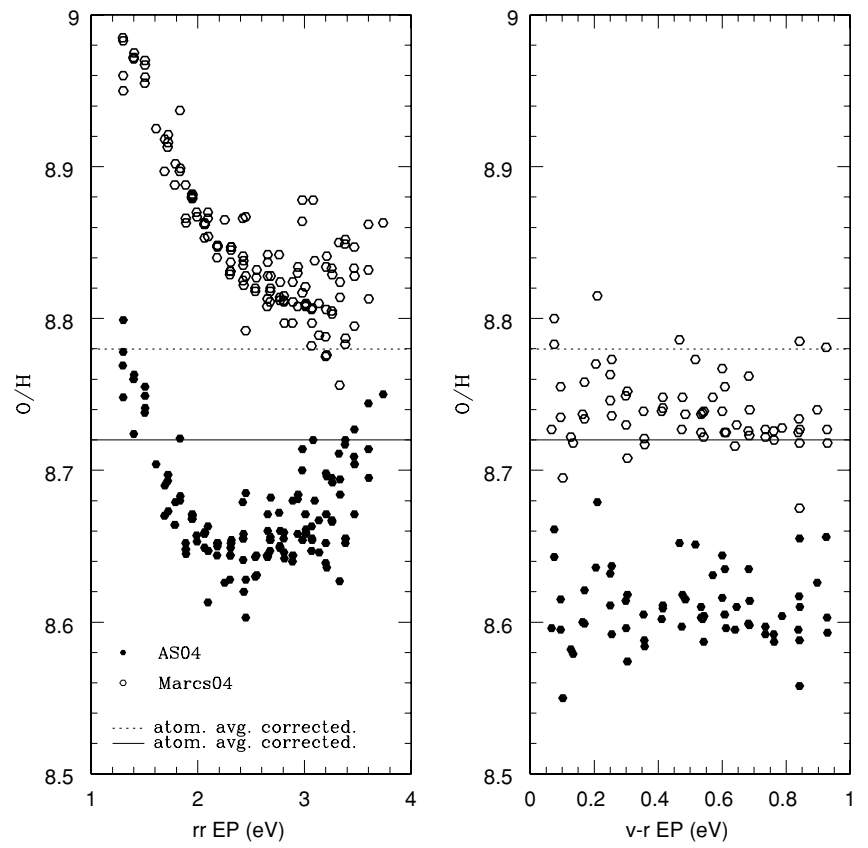

Figure 3. Oxygen abundances derived from infrared $\mathrm{OH}$ lines in the one-dimensional MARCS and three-dimensional AGSAK04 atmospheres are compared as a function of excitation potential. The left panel represents $(r, r)$ transitions, while the right panel represents (v,r) transitions. Results from the three-dimensional model atmospheres are solid circles; results from the MARCS theoretical one-dimensional model atmosphere are open circles. The solid and dashed lines indicate the mean abundances that would be derived from atomic features in the three-dimensional and MARCS models. The MARCS model is more internally consistent than the three-dimensional case, but similar trends with excitation potential are seen in both. Since the underlying model atmosphere code is similar, we conclude that the origin of these trends is in the basic model atmospheres rather than being induced by the treatment of convection.

than the one predicted by the simulations. Figure 1 suggests an alternate explanation, namely that the thermal structure in the outer layers is closer to the hotter semi-empirical HM74 model. We will return to this point when we consider more recent work on $\mathrm{CO}$ abundances in the outer solar atmosphere.

AGSAK04 discarded the $(r, r)$ data for the weaker and stronger lines, in effect deriving an abundance from the valley in Figures 3 and 4 . There is no better justification for discarding the high than the low points in this figure; such a procedure is not required for the one-dimensional HM models. We therefore derived average abundances using all of the features for all three models and both indicators; the standard deviation about the mean is an indicator of the quality of the fit for the individual bands. Our results are summarized in Table 1 . The average molecular abundances were obtained with a weighted mean, but a simple averaging of the errors underestimates the dispersion. We therefore computed $\sigma$ for each band around the weighted molecular mean in Table 1 and averaged the $(v, r)$ and $(r, r)$ values to obtain the total error in the molecular abundances presented there.

Meléndez (2004) considered a third molecular oxygen abundance indicator, and derived relative abundance patterns comparable to what AGSAK04 found. We do not include this in Table 1 because it is not clear that systematic errors between model atmospheres codes can be properly accounted for in a differential analysis. If we had included the Meléndez (2004), the mean molecular abundances would have been minimally altered for 3D and HM. The average would be reduced for MARCS and the internal error in the MARCS $[\mathrm{O} / \mathrm{H}]$ would be dramatically increased. This provides further evidence that there is an underlying issue in the thermal structure of the MARCS model. 


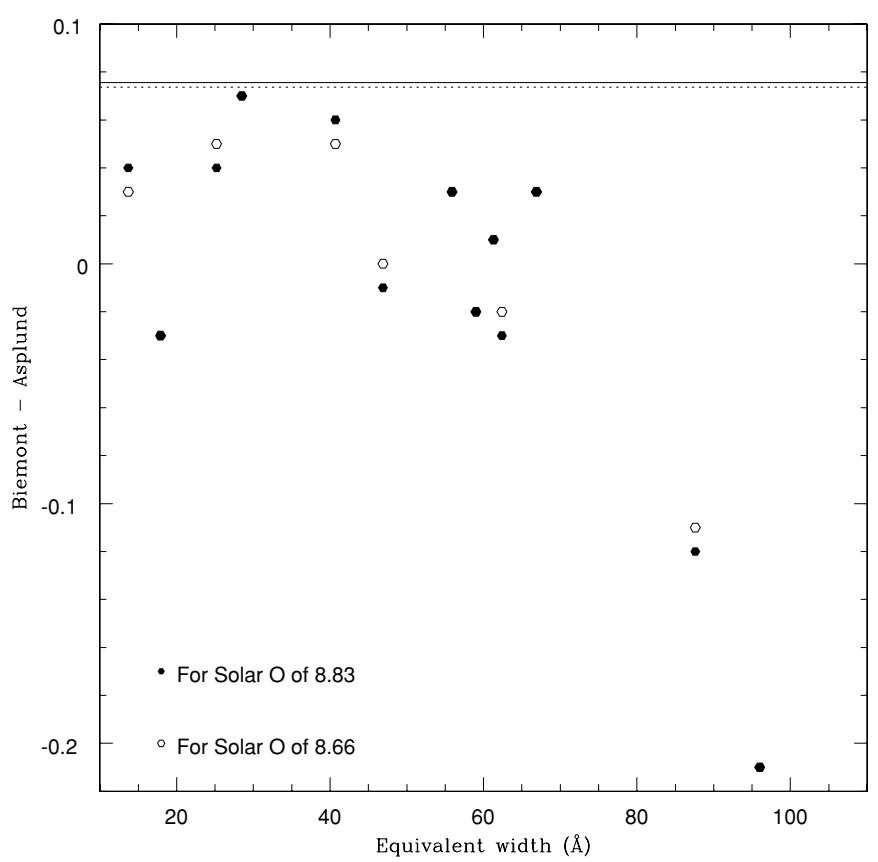

Figure 4. Comparison of the derived carbon abundance from the atomic lines common to Asplund et al. (2005a) and Biemont et al. (1993) as a function of the equivalent width. The differences between these abundances are in the sense of (Biémont-Asplund). Two cases ( $\log \epsilon_{O}=8.66$ and 8.83) have been plotted The data from Biemont et al. (1993) have been corrected for the difference in $\log (g f)$ and in equivalent width. The rms for the two cases is plotted as a solid line for the 8.83 case and as a dashed line for the 8.66 case.

Melendez also computed abundances with the same indicators as AGSAK04 for a Kurucz model atmosphere, and derived similar abundances as would be found for the HM74 model. Ayres et al. (2006) present evidence for a high solar oxygen derived from $\mathrm{CO}$ studies; we postpone a discussion of this interesting result to our conclusion, in the context of tests of the solar thermal structure (see also Scott et al. 2006).

\subsubsection{Oxygen Abundance and Error Analysis}

Our overall result from the reanalysis of the AGSAK04 oxygen indicators is that the abundances derived from the atomic indicators are systematically increased for all models. In the original paper, the three-dimensional abundance estimators were found to yield consistent abundances, while the one-dimensional abundances from different methods were highly discordant. This conclusion no longer holds when the reduced NLTE corrections inferred from limb-darkening studies are employed. In fact, one would obtain very similar conclusions to those presented in Table 1 from the triplet abundance of 8.72 presented in Allende Prieto et al. (2004) for the three-dimensional model.

Rather than simply adopting one model or another as correct, we interpret the difference between the HM74 and threedimensional abundances as evidence that the thermal structure of the Sun is intermediate between the two. The difference between the atomic and molecular abundances is roughly equal in magnitude and opposite in sign between these models; the MARCS model yields an intermediate abundance where the two classes of indicators give the same abundance, but with a larger error. The error in (atomic-molecular) is substantial; these differences are formally significant only at slightly more than $1 \sigma$. We therefore argue that the mean of the derived atomic abundances (8.75) is a reasonable estimator of what one would obtain from a model with a thermal structure capable of reproducing the atomic and molecular data; one would obtain 8.73-8.74 from comparing HM and three dimensions, and 8.76 from interpolating between HM and MARCS for obtaining a consistent atomic and molecular scale. We have a random error of 0.05 dex for the mean atomic abundance, but this is insufficient for a total error because of the presence of strong systematic differences. Adopting the consistency between atomic and molecular indicators as a measure of goodness of fit, $1 \sigma$ deviations could make either the threedimensional model $([\mathrm{O} / \mathrm{H}]=8.68)$ or the $\mathrm{HM}$ model $(8.80)$ consistent. We treat this as a $1 \sigma=0.06$ systematic error, and note that it is comparable to the zero-point shift that we obtain for the atomic indicators relative to AGSAK04 estimated below.

We can also examine systematic errors by comparing the AGSAK04 values with abundance estimates by other authors. These are most easily analyzed by comparing LTE abundances for the triplet. Holweger (2001) derived an average LTE triplet abundance of 8.78 for his standard model and 8.85 for the alternate VAL model. Biemont et al. (1991) reported 8.84 for the triplet for a HM model and 8.78 for a MACKKL atmosphere. These should be compared with $8.89,8.87$, and 8.93 for the three LTE cases in AGSAK04. We note that the one-dimensional cases in AGSAK04 used the equivalent widths obtained with the line broadening of the three-dimensional hydro models, so there will be differences between their results and those obtained with other one-dimensional codes. The average LTE abundance is 8.85 , with $\sigma=0.055$ dex. Although not rigorous, this exercise suggests that systematic differences at the 0.06 dex level are a reasonable estimate of the current state of the art for oxygen abundances when estimated with different techniques.

Adding systematic (0.06) and random (0.05) errors in quadrature, we obtain $8.75 \pm 0.08$ as our final oxygen abundance estimate for the Sun. This is less than $1.6 \sigma$ below the helioseismic abundance, and therefore we conclude that the existence of a solar oxygen problem has not been demonstrated with high statistical significance. Our final central value and error estimate are close to the Caffau et al. (2008) result of $8.76 \pm 0.07$ obtained after this paper was submitted.

\subsection{Carbon Abundance Indicators}

The overall story for carbon follows a similar path to the changes in the inferred oxygen abundance, and the comprehensive reanalysis of AGSAB05 for carbon has a similar logical structure to the 2004 oxygen paper. Although both the carbon and oxygen are reduced, the $\mathrm{C} / \mathrm{O}$ ratio is preserved. A cool and inhomogeneous outer solar atmosphere in the three-dimensional models yields substantially reduced abundances from molecular indicators, while blending features and NLTE effects reduce the carbon abundance inferred from atomic features. What distinguishes the carbon from the oxygen case is that in the case of oxygen there was a substantial NLTE correction, while the NLTE effects are smaller for carbon. As a result one might anticipate a smaller relative reduction in the carbon abundances inferred from atomic lines than the change in oxygen abundance inferred from atomic indicators. However, this is not the actual published result; if anything, the relative change in the derived carbon from atomic features is lower than the molecular value for all of the models presented in AGSAB05. Unlike the case of oxygen, this effect cannot be explained by any of the ingredients used to explain the differences in the comparison with prior work given by AGSAB05. Until the origin of this difference is understood, we therefore have to consider two different systematic sets of abundances for atomic features, and 
Table 3

Comparison of the Different C Abundances Derived from the Indicators Used by Asplund et al. (2005b) When Different Sets of Correction are Applied

\begin{tabular}{|c|c|c|c|c|c|c|}
\hline Indicator & Mean & Error & Mean & Error & Mean & Error \\
\hline & $3 \mathrm{D}$ & & HM & & M & \\
\hline$[\mathrm{C} \mathrm{I}]$ & 8.39 & 0.04 & 8.45 & 0.04 & 8.40 & 0.04 \\
\hline $\mathrm{C}_{\mathrm{I}}$ & 8.36 & 0.03 & 8.39 & 0.04 & 8.35 & 0.03 \\
\hline $\mathrm{CH}$-vib-rot & 8.38 & 0.04 & 8.53 & 0.04 & 8.42 & 0.04 \\
\hline C2 electronic & 8.44 & 0.03 & 8.53 & 0.03 & 8.46 & 0.03 \\
\hline $\mathrm{CH}$ electronic & 8.45 & 0.04 & 8.59 & 0.04 & 8.44 & 0.04 \\
\hline Average & 8.39 & 0.04 & 8.48 & 0.04 & 8.40 & 0.04 \\
\hline Deviation & 0.03 & & 0.07 & & 0.05 & \\
\hline ATM & 8.37 & & 8.42 & & 8.37 & \\
\hline MOL & 8.42 & & 8.54 & & 8.44 & \\
\hline ATM-MOL & -0.05 & & -0.12 & & -0.07 & \\
\hline Corrected Low Atomic Scale & $3 \mathrm{D}$ & & $\mathrm{HM}$ & & M & \\
\hline$[\mathrm{C}$ I $]$ & 8.39 & 0.04 & 8.45 & 0.04 & 8.40 & 0.04 \\
\hline $\mathrm{C}_{\mathrm{I}}$ & 8.40 & 0.03 & 8.44 & 0.04 & 8.40 & 0.03 \\
\hline $\mathrm{CH}$-vib-rot & 8.38 & 0.04 & 8.53 & 0.04 & 8.42 & 0.04 \\
\hline $\mathrm{C} 2$ electronic & 8.44 & 0.03 & 8.53 & 0.03 & 8.46 & 0.03 \\
\hline $\mathrm{CH}$ electronic & 8.45 & 0.04 & 8.59 & 0.04 & 8.44 & 0.04 \\
\hline Average & 8.41 & 0.04 & 8.49 & 0.04 & 8.42 & 0.04 \\
\hline Deviation & $\mathbf{0 . 0 3}$ & & 0.06 & & $\mathbf{0 . 0 3}$ & \\
\hline ATM & 8.40 & & 8.45 & & 8.40 & \\
\hline MOL & 8.42 & & 8.54 & & 8.44 & \\
\hline ATM-MOL & $-\mathbf{0 . 0 2}$ & & -0.09 & & -0.04 & \\
\hline Corrected high Atomic Scale & $3 \mathrm{D}$ & & HM & & $\mathrm{M}$ & \\
\hline$[\mathrm{C} \mathrm{I}]$ & 8.43 & 0.04 & 8.49 & 0.04 & 8.44 & 0.04 \\
\hline $\mathrm{C}_{\mathrm{I}}$ & 8.48 & 0.03 & 8.52 & 0.04 & 8.48 & 0.03 \\
\hline $\mathrm{CH}$-vib-rot & 8.38 & 0.04 & 8.53 & 0.04 & 8.42 & 0.04 \\
\hline C2 electronic & 8.44 & 0.03 & 8.53 & 0.03 & 8.46 & 0.03 \\
\hline $\mathrm{CH}$ electronic & 8.45 & 0.04 & 8.59 & 0.04 & 8.44 & 0.04 \\
\hline Average & 8.44 & 0.04 & 8.59 & 0.04 & 8.45 & 0.04 \\
\hline Deviation & 0.04 & & 0.04 & & 0.02 & \\
\hline ATM & 8.46 & & 8.50 & & 8.46 & \\
\hline MOL & 8.42 & & 8.54 & & 8.44 & \\
\hline ATM-MOL & 0.04 & & -0.04 & & 0.02 & \\
\hline
\end{tabular}

Notes. The top panel corresponds to the results derived in Asplund et al. (2005b), the middle panel corresponds to the revised value when we adopt another NLTE correction that is smaller than those of Asplund et al. (2005b), and the bottom panel includes a shift of $+0.035 \mathrm{dex}$ and $+0.08 \mathrm{dex}$ in the forbidden and allowed lines, respectively (see the text for detail).

the best choice of model hinges on which set is correct. In this section, we discuss the three classes of indicators (forbidden and allowed atomic lines, and molecular) in turn, and as for the oxygen abundance synthesize our final best estimate and error in the fourth subsection. Our overall estimates are presented in Table 3. The top set of values represents the original AGSAB05 values for the different indicators. The middle set is what we would obtain with the low AGSAB05 normalization of the atomic abundances, while the bottom set is what we obtain with the higher Biemont/Holweger normalization.

\subsubsection{Forbidden Lines}

The [C I] line at $8727 \AA$ was the subject of a detailed analysis by Allende Prieto et al. (2002). They incorporated blending from a nearby $\mathrm{Si}$ feature to reduce the equivalent width attributable to carbon from 6.5 to $5.3 \mathrm{~m} \AA$, with a corresponding reduction in the inferred abundance. They also employ lower oscillator strength than previous studies. Unlike the case of oxygen, the carbon abundance derived from the forbidden line is somewhat temperature sensitive as that derived from molecular features, so the atomic versus molecular diagnostic is less powerful for carbon than for oxygen. We adopt the AGSAB05 central values for our base case, but note that there may be explained systematics in the atomic carbon abundances in AGSAB05 which we discuss below. We include their error estimate for uncertainties in the equation of state $(0.02 \mathrm{dex})$, but assign a larger uncertainty to the atomic physics $(0.04 \mathrm{dex})$ in accord with the quoted theoretical uncertainties. Our principal reservation on the error budget is the uncertainty in the continuum level and the contribution to the equivalent width of the blend from the wing of the Si feature. Their reduced $\chi^{2}$ permits only small deviations (of order $0.01 \mathrm{dex}$ ) in the derived carbon abundance, but the base model relies upon the assumption that the underlying velocity field is exact. Although the overall agreement with Fe (Asplund et al. 2000b) and Si (Asplund 2000) line profiles is good, it is not errorless. We cannot evaluate this ingredient directly, but an estimate based upon the mean deviation observed in clean lines would seem to be a worthwhile exercise. For the present, we therefore assign the same blending uncertainty of 0.03 dex adopted by Allende Prieto et al. (2002) to obtain a total uncertainty of 0.054 dex.

\subsubsection{Permitted Atomic Lines}

AGSAB05 considered a subset of the permitted atomic features used in previous solar abundance studies (e.g., Biemont et al. 1993; Stuerenburg \& Holweger 1990). Stuerenburg \& Holweger (1990) found that small NLTE corrections are required for CI when including hydrogen collisions, and that the strength of the correction depends on the equivalent width. They found an average of $-0.05 \mathrm{dex}$; if restricted to the weaker lines included in AGSAB05, their average NLTE correction would be -0.02 dex. AGSAB05 computed NLTE corrections for onedimensional models, and the MARCS corrections were applied to the three-dimensional models. Hydrogen collisions were not included in the NLTE corrections; this resulted in larger downward abundance revisions (an average of -0.07 to -0.08 ) than Stuerenburg \& Holweger (1990). AGSAB05 note that the case of carbon should be an analog of oxygen, and we concur. As a result, we contend that the case with hydrogen collisions should be included in the base model. Both sources indicate that including hydrogen collisions roughly halves the expected NLTE correction. We therefore considered two cases for NLTE corrections: a maximum of half the AGSAB05 value (corresponding to their hydrogen collision case, average $-0.04 \mathrm{dex}$ ) and a minimum of one quarter of the AGSAB05 value (corresponding to the SH90 case, average of $-0.02 \mathrm{dex}$ ). Our best value is the average between the two (a mean of $-0.03 \mathrm{dex}$ ), and the error induced by uncertainties in NLTE corrections is 0.01 dex; adopting the AGSAB05 hydrogen collision case would only have changed our mean value by $0.01 \mathrm{dex}$. We applied these proportional NLTE corrections to the AGSAB05 LTE results for their three classes of models (middle values, Table 3 ). There is a small reduction in the dispersion (and mean trend with equivalent width) for the one-dimensional models and a corresponding increase for both in the three-dimensional model; none of these features, however, are drastic. The internal dispersion in the permitted atomic abundances is of order 0.03 dex.

A more substantial issue emerges when we compare the AGSAB05 abundances with prior work, and this is true even for the LTE estimates. The mean LTE abundance for the Biemont et al. (1993) sample is 8.56 for the lines in common with AGSAB05; this should be compared with a HM LTE value of 8.48 for the latter compilation. This 0.08 dex offset is com- 
parable to the average difference between atomic and molecular abundance indicators. The mean difference in equivalent width and oscillator strength for the lines in common is negligible, and would yield an offset of less than 0.01 dex from a curve of growth analysis (J. Johnson 2008, private communication). We illustrate the differences in Figure 4, defined in the sense (Biemont-AGSAB05). In this figure, we have corrected the Biemont abundances to the AGSAB05 equivalent width and oscillator strengths. The differences are significant even for weak lines, suggesting that differences in the classical line broadening are probably not responsible. A similar, but smaller, effect is present in the forbidden line. Allende Prieto et al. (2002) inferred a HM abundance of 8.48, which would also be obtained from Stuerenburg \& Holweger (1990) when a blending correction is made to the equivalent width. AGSAB05 could not trace a comparable difference ( $0.06 \mathrm{dex})$ relative to the earlier work of Lambert. The only obvious source that we can derive is a note by Sturenburg and Holweger that they corrected their atomic abundances for the fraction of $\mathrm{C}$ tied up in $\mathrm{CO}$, which could be of the right order to explain the differences. It is hardly surprising that complex abundance studies have systematics at this level, but by the same token precise abundance claims demand such a degree of precision. Until the origin of this discrepancy (which is not present for oxygen) is explained, we have to treat this as a systematic uncertainty in the atomic abundance scale. Abundances derived under this scale are the last set of values in Table 3.

\subsubsection{Molecular Lines}

We consider the same four molecular indicators that were included in AGSAB05. They chose to disregard one of them ( $\mathrm{CH}$ electronic lines) in their derived mean abundances, on the ground that they are located in a crowded portion of the spectrum and sensitive to the treatment of line broadening. However, the formal errors in the $\mathrm{CH}$ electronic abundances are similar to those for the other molecular species, and as such we see no obvious reason to exclude them. We do treat the $\mathrm{CH}(\mathrm{v}, \mathrm{r})$ abundances as being more reliable, as they are based on many more lines than the other diagnostics. We therefore assigned double weight to the $\mathrm{CH}$ values and single weight to both the $\mathrm{C} 2$ electronic and $\mathrm{CH}$ electronic values. As for oxygen, the mean was derived by a weighted average of the carbon obtained with different molecular indicators, and the scatter of the individual line measurements for all diagnostics around the adopted mean was taken as a measure of the random error. We did not include carbon (or oxygen) abundances derived from CO line studies, because there are complex correlated errors. Had we included them, the net effect would have been to increase the molecular abundances relative to the atomic values.

\subsubsection{Carbon Abundance and Error Analysis}

Our final inference concerning carbon depends on which atomic abundance scale is adopted. If we take the low scale of AGSAB05, the three-dimensional model atomic and molecular abundances $(8.40,8.42)$ are closer than those for HM74 $(8.45$, 8.55); one-dimensional MARCS abundances are also consistent $(8.40,8.44)$. We would estimate a mean value of $8.41-8.42$, with a random error of 0.04 dex. The HM74 average of 8.50 would be an effective $2 \sigma$ internal inconsistency, implying a 0.04 dex systematic uncertainty for a total abundance of $8.41 \pm 0.06$. Adopting the higher scale would give pairwise results of (8.44, $8.42),(8.50,8.55),(8.44,8.44)$; all three models are internally consistent within the errors, and a mean abundance would be
8.47 with a total error of 0.05 ( 0.04 random, 0.03 systematic). We adopt the mean of these approaches (8.44), and estimate an error of 0.04 (random) and 0.04 (systematic) for a total of 0.06 dex when combined in quadrature.

\subsection{Nitrogen Abundance Indicators}

Our discussion of nitrogen is necessarily briefer than that of oxygen and carbon, largely because the published results are preliminary and incomplete. Holweger (2001) derived a NLTE $[\mathrm{N} / \mathrm{H}]=8.0 \pm 0.11$, comparable to results in previous compilations of solar abundances from Grevesse \& Sauval (1998). The compilation of models in Asplund et al. (2005a) yields atomic and molecular nitrogen abundance estimates of $(7.85 \pm 0.08,7.73 \pm 0.05),(7.97 \pm 0.08,7.95 \pm 0.05),(7.94 \pm$ $0.08,7.82 \pm 0.05)$ for $3 \mathrm{D}, \mathrm{HM}$, and MARCS, respectively. The same correspondence between three dimensions and MARCS that was seen in oxygen is replicated in nitrogen, but the internal consistency in the HM model is higher than that in the other models. The formal significance of the disagreement in the three-dimensional models is under $2 \sigma$, however, so we cannot exclude the possibility that they may be consistent. We therefore adopt the HM result as the central value $(7.96 \pm 0.06)$, and treat the difference with the three-dimensional result $(7.78 \pm 0.06)$ as a $2 \sigma$ systematic error. This yields a total uncertainty in $[\mathrm{N} / \mathrm{H}]$ of 0.10 dex dominated by systematic uncertainties. The most recent nitrogen abundance study, that of Caffau et al. (2009), recommends $\mathrm{N} / \mathrm{H}=7.86 \pm 0.12$ from their three-dimensional simulations.

\subsection{The Solar Beryllium Abundance}

There is an interesting linkage between the solar $\mathrm{O}$ and Be abundances. In stellar interiors, Be is destroyed at modest temperatures (of order 3.5 million $\mathrm{K}$ ). It can therefore be used as a diagnostic of mixing in stars, especially in conjunction with the more fragile light element Li (Pinsonneault 1997). Traditional model atmosphere studies (Chmielewski et al. 1975) yield a solar photospheric beryllium abundance roughly half of the meteoritic abundance. However, the only accessible Be feature is located in a crowded portion of the spectrum in the near UV, and the continuum opacity is uncertain in this regime (largely from the contribution of numerous weak iron lines). Since the strength of a line is a function of the ratio of the line to the continuous opacity, a higher photospheric Be could be derived if the continuous opacity background was higher than that of the model. In an important paper, Balachandran \& Bell (1998) pointed out that nearby OH lines could be used to test the continuous opacity close to beryllium. They derived a UV OH lines that were too strong if they used the absolute oxygen abundance obtained from the IR OH lines, and interpreted this as evidence that the continuous opacity is underestimated in the spectral window relevant for Be. Similar conclusions for three-dimensional models were obtained by Asplund (2004). Following Lodders (2003), we note that the uncertainties in the ad hoc corrections are substantial. Asplund (2004) quotes photospheric and meteoritic abundance errors of 0.09 and 0.08 dex respectively, implying that his zero net photospheric depletion has a $1 \sigma$ uncertainty of 0.12 dex. Even if the Balachandran and Bell argument is entirely correct, these data set a $2 \sigma$ limit of 0.24 dex on beryllium depletion and do not require that it be zero. There is also the possibility of substantial NLTE corrections to the UV OH lines, which would reduce or even eliminate the requirement for a mechanism to 
reduce the strength of the lines. We also note that the value of the oxygen used by Balachandran \& Bell (1998) for the IR lines (8.91) in the HM74 model is larger than the value derived from other molecular and atomic indicators, and even slightly larger than the value from the (v,r) transitions in the same model from the work of Asplund and collaborators. We contend that this promising approach still has substantial errors, including large uncertainties in the absolute solar oxygen abundance. We therefore believe that the approach of Lodders (2003) is the best current picture of the degree of beryllium depletion in the Sun: namely, there is a substantial uncertainty in the degree of solar beryllium depletion, and that further work is required before powerful observational bounds can be used to constrain interiors calculations.

\section{CONCLUSIONS AND FUTURE TESTS}

Our basic conclusion is simple: the difference between the solar CNO abundances as derived from model atmospheres and model interiors considerations is not statistically significant. The systematic errors in photospheric abundance indicators will have to be reduced before a "solar abundance problem" can be established (or ruled out) with confidence. However, the disagreement between the solar thermal structure and that of the simulations would favor the higher abundance scale, and there is some recently published evidence to that effect. If this is confirmed, it switches the nature of the problem from being a question of the correct abundance scale to a question of the uncertainties in numerical convection simulations. We begin with a synthesis and explanation of our findings. We then divide our conclusions into two parts. We recommend steps to more firmly establish the photospheric abundance scale, and contend that accurate solar abundances require tests of the thermal structure of the models and the magnitude of NLTE abundance corrections. In our final subsection we then gather together evidence that the atmospheric abundance scale problem may be tied to the limited resolution in the convection simulations or errors in the underlying model atmosphere treatment. The consequences for the solar beryllium abundance, which is a useful diagnostic of internal mixing, are also explored.

The two main justifications for the superiority of the threedimensional hydro atmospheres are the treatment of line broadening and the inclusion of granulation. Both of these represent genuine improvements in the atmospheric physics. However, neither of these effects is actually primarily responsible for the difference in the solar abundance scale. Many of the abundance indicators are insensitive to the effective microturbulence. If temperature fluctuations are imposed on a semi-empirical Holweger-Mueller atmosphere, the resulting granulation corrections are usually smaller than the three-dimensional convection effects reported by Asplund and collaborators, and frequently opposite in sign (Holweger 2001). Very similar trends are present in the three-dimensional models of Caffau et al. (2008), which have a thermal structure closer to the HolwegerMuller atmosphere than was the case for the Asplund models. The main driver behind the systematic reductions in abundance derived from AGSAK04 relative to GS98 is a theoretically predicted change in the thermal structure, coupled with large assumed NLTE corrections for atomic features. Neither of these changes is directly supported by observational tests. Instead, the argument for the superiority of the abundances derived from the newer model atmospheres is an indirect one, focused on the concordance of abundances derived from different indicators.
A consistent chain of logic emerges from the comprehensive studies of oxygen (AGSAK04) and carbon (AGSAB05). Classical LTE model atmospheres tend to yield internally consistent, and high, carbon and oxygen abundances for atomic and molecular indicators. The application of a different thermal structure in the three-dimensional hydro atmospheres drastically reduces the abundances inferred from highly temperature sensitive molecular indicators, but has a smaller effect on atomic features. Large NLTE corrections are then applied to the abundances derived from permitted atomic features for both one-dimensional and three-dimensional models. The net result is that the abundant estimates from one-dimensional models become internally inconsistent (atomic indicators yield lower abundances than molecular ones), while abundances derived from the three-dimensional models are internally consistent. The abundances derived from forbidden lines are insensitive to NLTE effects, but they are reduced in the newer generation of models by the inclusion of blending features. As a secondary argument, the fits to individual indicators are argued to be superior in the three-dimensional models when compared to the fits to individual indicators in the one-dimensional models. This approach is appealing on the surface, but when examined in detail the picture is decidedly more ambiguous. If anything, the hints from the data would lean toward the opposite conclusion.

The abundances derived from forbidden lines have the smallest systematic errors, but errors in both the theoretical oscillator strengths and the treatment of blending features result in nonnegligible random errors. More to the point, the internal consistency of abundances derived from forbidden and molecular lines is actually similar in the three-dimensional and one-dimensional cases. From Table 1, the forbidden and molecular oxygen abundances are $(8.70,8.63)$ for $3 \mathrm{D}$ and $(8.78,8.84)$ for HM; the differences are almost identical. Given the errors, neither discrepancy is statistically significant.

The abundances reported for permitted atomic features in AGSAK04 and AGSAB05 are significantly lower for onedimensional models than the corresponding molecular abundances, while the reported three-dimensional results are in agreement. In the case of oxygen, this rests completely on the assignment of large NLTE corrections. These corrections were obtained under the assumption that hydrogen collisions were unimportant. Detailed studies of the response of the triplet to limb darkening indicate that models including hydrogen collisions are favored, and the inferred NLTE corrections decrease. As a result, the internal consistency of the oxygen indicators is comparable for the different classes of atmospheres. Nitrogen is consistent for HM74 models and inconsistent (but at less than $2 \sigma$ ) for the three-dimensional case. In the case of carbon, the situation is made more complex by significant zeropoint offsets between earlier studies of carbon abundances that are not explained. Again, the assignment of larger NLTE corrections is uncertain (and, unlike the case of oxygen, not directly tested against limb-darkening data). A clean distinction between models on the basis of consistency is not obtained. However, the three-dimensional models do yield different molecular and atomic abundances for both $\mathrm{N}$ and $\mathrm{O}$, and might also do so for $\mathrm{C}$.

One might then hope to find distinct differences in the quality of the fits to different molecular indicators. The usual patterns, unfortunately, manifest themselves as simple zero-point shifts. For every case where there are issues with the one-dimensional models (e.g., small trends with excitation potential in the $[\mathrm{O} / \mathrm{H}]$ derived from $(\mathrm{v}, \mathrm{r}) \mathrm{OH}$ transitions in the $\mathrm{HM}$ model) there are 
comparable or larger effects for the three-dimensional models (e.g., substantial trends in the $[\mathrm{O} / \mathrm{H}]$ derived from $(\mathrm{r}, \mathrm{r}) \mathrm{OH}$ transitions). Scott et al. (2006) examined CO indicators, and the resulting pattern is illustrative. The three-dimensional models yielded similar results for two of the three features studied, while the one-dimensional models performed better in a different pair of indicators. The $\mathrm{C}^{12} / \mathrm{C}^{13}$ ratio from the one-dimensional models ranges from 69 to 84 , while the same ratio for the three-dimensional models ranges from 83 to 108 . These values should be contrasted with the expected terrestrial ratio of 89 . Scott et al. (2006) choose comparisons that favor the threedimensional models, while an advocate of the traditional models might reasonably stress the other cases. In our view, the best choice of models is not clearly distinguishable from the CNO abundance studies. We recommend caution when extrapolating these model results to other stars, where the differential effects can be even more drastic.

\subsection{Establishing the Absolute Photospheric Abundance Scale}

The single most important test that is required for atmospheric theory is a discriminant between the different proposed thermal structures of the solar atmosphere. The paper by Ayres et al. (2006) makes an important contribution by making direct comparisons of solar data with the thermal properties of the simulations. They present evidence that the solar center-tolimb variations in continuum flux are inconsistent with the predictions of the three-dimensional hydro simulations. They also note that the predicted magnitude of fluctuations in the upper atmosphere from the simulations appears to be larger than the observed pattern. Ayres et al. then construct an empirical model of the atmosphere and derive a high oxygen abundance (8.85) from $\mathrm{CO}$ molecular features under the assumption of a fixed $\mathrm{C} / \mathrm{O}$ ratio. In retrospect this conclusion is not surprising. The HM model is not a purely theoretical exercise; it was constructed to reproduce the mapping of the source function as a function of optical depth inferred from limb-darkening studies of continuum flux and strong lines (see also Allende Prieto et al. 1998). The relative trends we have inferred from atomic and molecular abundance indicators support the conclusions of Ayres et al., but the current errors make our evidence in this matter suggestive but not conclusive. After this paper was submitted, Koesterke et al. (2008) performed such a detailed analysis. They found that the one-dimensional averaging of the three-dimensional model features exaggerated the underlying differences with the model atmospheres. However, the threedimensional models did not reproduce the limb darkening in the continuum, with deviations comparable to those of purely theoretical one-dimensional models. This confirms the basic finding of Ayres et al. (2006).

Ultimately, the absolute accuracy of photospheric abundances is directly tied to the absolute accuracy of the thermal structure. This suggests that an approach similar to that of Steffen \& Holweger (2002) may be the optimal one. In their paper they examined the impact of temperature fluctuations around an assumed mean empirical thermal structure, which in their case was the HM74 model. Interestingly, the abundance corrections that they derive would act in the sense of increasing the concordance between abundance indicators. Oxygen abundances from atomic indicators would be slightly increased; although they did not consider molecular features directly, the net effect would certainly have the same sign as that obtained from three-dimensional hydro models, namely a decrease in the inferred abundance. In such a differential approach, deviations between the mean structure of the simulations and the empirical data would be used as guidance concerning the underlying physics. In contrast, the three-dimensional model abundances assume that the ab initio profile is correct. A similar approach could be employed for the velocity field that replaces the microturbulence and macroturbulence in traditional one-dimensional atmospheres.

A second ingredient that must be tested empirically, rather than by theoretical assertion, is the magnitude of NLTE corrections. The available evidence suggests that NLTE corrections are in general small for the Sun, but for the level of precision required in the absolute abundance scale these small corrections are significant. Studies of different spectral features yield different conclusions about the physical model employed in NLTE studies. This implies that there are significant uncertainties in absolute theoretical calculations. Fortunately, NLTE corrections can be constrained by the response of line strength to limb darkening in the Sun. It should be possible to develop improved theoretical models with a sufficient database of information developed in this fashion. One other stringent test of NLTE effects may be to focus on the species whose relative abundances can be reliably inferred from meteoritic data. For example, NLTE effects may be significant for iron (Shchukina \& Trujillo Bueno 2001) but less so for Si (Wedemeyer 2001). Holweger (2001) noted that there may be a conflict between the photospheric and meteoritic $\mathrm{Fe} / \mathrm{Si}$ ratio, albeit one of the marginal significance. A similar situation may exist for $\mathrm{Na}$ (Asplund et al. 2005a).

Another tractable problem is the absolute error for the forbidden $\mathrm{C}$ and $\mathrm{O}$ lines. In these cases, uncertainties in the line profiles and continuum levels should be included. Better atomic data (such as oscillator strengths for both the lines and the blending features) would also be useful. As the Caffau et al. (2008) work has made clear, there also remain significant judgement calls to be made in the choice of solar data, continuum levels, and treatment of blending features.

The accuracy of the theoretically predicted turbulent velocity field as a function of optical depth should also be subjected to a more rigorous analysis. Scott et al. (2006) present evidence that the generation of simulations used for the abundance analysis yielded poor fits to the line bisectors of $\mathrm{CO}$ lines, although improved over one-dimensional predictions. It is worth keeping in mind that line profiles are integral quantities, and as a result the uniqueness of the solutions is not established by individual cases of good fits. This is particularly true when the abundance itself is treated as a free parameter. It would be extremely useful if future papers on abundances derived using numerical simulations illustrated individual line fits, as well as quantifying the actual impact of the "effective microturbulence" on the abundance estimates.

It is useful to separate out the impact of velocity broadening from the effect of granulation and temperature gradient changes. This can be done by using the mean thermal structure and temperature fluctuations from the simulations and a more traditional micro/macroturbulence model to infer abundances, and comparing the results with the full three-dimensional models. Scott et al. (2006) constructed such a test case (their one-dimensional AV model), and found only small abundance offsets, of order 0.04 dex for oxygen derived from IR OH lines. They also inferred carbon abundances from $\mathrm{CO}$; in this case $\mathrm{O}$ was held fixed and the carbon was adjusted to fit different molecular indicators. The deviations in the derived carbon abundances relative to the three-dimensional case ranged from 
small (0.01 dex for the LE lines) to modest (0.06 dex for the weak $\Delta v=1$ lines $)$ to large $(0.14$ dex for the $\Delta v=2$ lines $)$. These deviations may explain the changes in excitation potential that Ayres et al. (2006) needed to obtain consistent abundances within a one-dimensional framework. This exercise implies that the impact of the improved microphysics varies substantially for different indicators, and is worth quantifying across the board. An alternate exercise (using the revised velocity field and relative temperature fluctuations while adopting a HM74 mean thermal structure) might also illuminate.

\subsection{Uncertainties in Numerical Convection Simulations}

First-principles theoretical model atmosphere calculations have undeniable strengths. The ability to naturally reproduce line widths and include granulation is a powerful addition to our ability to reliably interpret stellar and solar spectra. The principal difficulty with such models is that errors in the input physics generate absolute errors in the inferred atmospheric structure that cannot be calibrated away in the absence of explicit free parameters. This phenomenon is the major reason why numerical convection simulations have not replaced the simple mixing length theory in stellar interiors calculations. Interiors models that can reproduce observed stellar radii are simply more useful for most purposes than models with a better physical treatment of convection that fails to do so.

Before the results from such models are adopted as the new abundance standard, it will be necessary to perform an extensive theoretical error analysis and to compare the models with the strongest observational constraints. We believe that accurate solar abundance calculations must reproduce the observed solar thermal structure, and from the Ayres et al. (2006) paper the Asplund models employing numerical convection simulations appear to yield a temperature gradient steeper than the real Sun. This could be caused by errors in the background (1D) stellar atmosphere treatment; for example, uncertainties in the equation of state and continuous opacities will induce absolute errors in the thermal structure. An approach similar to that employed in interiors models would be useful for assessing the uncertainties in the thermal structure and abundance predictions, and this should be included in the error budget for abundances.

It is more likely, however, that the major error source in three-dimensional hydro model atmospheres is related to uncertainties in the numerical convection simulations. The approximations in hydro simulations of giant planet atmospheres are demonstrated to be strongly affected by the quality of the assumed physics (Evonak \& Glatzmaier 2004). Zhang \& Schubert (2006) also provide a good summary of the uncertainties in the related problem of terrestrial and solar dynamo models. Another phenomenon that could be related is the issue of convective overshooting below surface convection zones. Numerical simulations have tended to favor extensive overshooting, and the early models had a substantial nearly adiabatic overshoot region, in conflict with the stringent limits set by seismology (less than $0.05 H_{p}$ ). More recent three-dimensional Brummell et al. (2002) and two-dimensional (Rogers \& Glatzmaier 2005; Rogers et al. 2006) calculations found that the filling factor for plumes is smaller than previously thought, which led to an overestimate in earlier models of the changes induced by overshooting in the thermal structure. The newer simulations predict strongly subadiabatic overshooting (effectively, overmixing without changing the thermal structure), which is consistent with the seismic limits. However, they still produce a substantial mixed region below the surface convection zone of order $0.4 H_{p}$.
Since even a small overmixing of $0.05 H_{p}$ drastically increases pre-MS lithium depletion (Pinsonneault 1997), which is already too efficient relative to stellar data (Piau \& Turck-Chièze 2002), it is likely that even this reduced overshooting is too large to be compatible with stellar constraints. We argue that there is a common pattern in both "undershooting" and "overshooting" above and below convective regions. In both cases, the numerical simulations may overestimate the degree of mixing and the impact on the thermal structure of convection outside the formal bounds set by the Schwartzschild criterion.

There are two plausible error sources that should be investigated. The treatment of heat transfer in the atmospheric convection simulations is necessarily simplified, and this may lead to an artificial inhibition in energy transport between turbulent cells projected into the radiative atmosphere and their surroundings. Resolution effects, however, may also be important. Even the highest resolution simulations available today are many orders of magnitude away from being able to reproduce the characteristic Reynolds numbers in the Sun. Scott et al. (2006) found significant changes in line bisectors for the outer layers of their solar model when they increased their resolution, and these changes were in the sense of reducing the temperature contrast in the upper atmosphere and improving the shape of the bisectors relative to data. Numerical tests with substantially increased resolution may shed some interesting light on the sensitivity of the predictions to the underlying numerics; two-dimensional convection simulations may be useful in this regard. The recent work by Caffau et al. (2008) provides a valuable independent test of atmospheric models. Their models employ more spectral resolution in the heat transport solution than the earlier AGSAK04 models did, and the resulting thermal structure is significantly closer to empirical limb-darkening data. We are optimistic that the net effect of such testing will be a greatly improved understanding of the strengths and weaknesses of theoretical atmospheric models, just as we are confident that the net result of the solar abundance controversy will be a far more secure knowledge of stellar abundances.

We thank Martin Asplund for providing tables of the abundances derived from molecular oxygen abundance indicators. We also thank Don Terndrup, Jennifer Johnson, Andreas Korn, Chris Sneden, and Hans Ludwig for helpful discussions on stellar abundance determinations. F.D. thanks Claude Zeippen for discussions on the uncertainties in the atomic data. M.H.P. acknowledges partial support from DOE-NNSA grant Number NA29580.

\section{REFERENCES}

Allende Prieto, C., Asplund, M., \& Fabiani Bendicho, P. 2004, A\&A, 423, 1109 Allende Prieto, C., Lambert, D. L., \& Asplund, M. 2001, ApJ, 556, L63 Allende Prieto, C., Lambert, D. L., \& Asplund, M. 2002, ApJ, 573, L137 Allende Prieto, C., Ruiz Cobo, B., \& Garcia Lopez, R. J. 1998, ApJ, 502, 951 Anders, E., \& Grevesse, N. 1989, Geochim. Cosmochim. Acta, 53, 197 Antia, H. M., \& Basu, S. 2006, ApJ, 644, 1292

Asplund, M. 2000, A\&A, 359, 755

Asplund, M. 2004, A\&A, 417, 769

Asplund, M., Grevesse, N., \& Sauval, A. J. 2005a, in ASP Conf. Ser. 336, Cosmic Abundances as Records of Stellar Evolution and Nucleosynthesis, ed. T. G. Barnes III \& F. N. Bash (San Francisco, CA: ASP), 25

Asplund, M., Grevesse, N., Sauval, A. J., Allende Prieto, C., \& Kiselman, D. 2004, A\&A, 417, 751

Asplund, M., Grevesse, N., Sauval, A. J., Allende Prieto, C., \& Blomme, R. 2005b, A\&A, 431, 693

Asplund, M., Grevesse, N., Sauval, A. J., Allende Prieto, C., \& Kiselman, D. 2005c, A\&A, 435, 339 
Asplund, M., Nordlund, Å., Trampedach, R., Allende Prieto, C., \& Stein, R. F. 2000a, A\&A, 359, 729

Asplund, M., Nordlund, Å., Trampedach, R., \& Stein, R. F. 2000b, A\&A, 359, 743

Ayres, T. R. 2008, ApJ, 686, 731

Ayres, T. R., Plymate, C., \& Keller, C. U. 2006, ApJS, 165, 618

Badnell, N. R., Bautista, M. A., Butler, K., Delahaye, F., Mendoza, C., Palmeri, P., Zeippen, C. J., \& Seaton, M. J. 2005, MNRAS, 360, 458

Balachandran, S. C., \& Bell, R. A. 1998, Nature, 392, 791

Barklem, P. S. 2007, A\&A, 466, 327

Basu, S., \& Antia, H. M. 2004, ApJ, 606, L85

Biemont, E., Hibbert, A., Godefroid, M., \& Vaeck, N. 1993, ApJ, 412, 431

Biemont, E., Hibbert, A., Godefroid, M., Vaeck, N., \& Fawcett, B. C. 1991, ApJ, 375,818

Brummell, N. H., Clune, T. L., \& Toomre, J. 2002, ApJ, 570, 825

Caffau, E., Ludwig, H.-G., Steffen, M., Ayres, T. R., Bonifacio, P., Cayrel, R., Freytag, B., \& Plez, B. 2008, A\&A, 488, 1031

Caffau, E., Maiorca, E., Bonifacio, P., Faraggiana, R., Steffen, M., Ludwig, H.-G., Kamp, I., \& Busso, M. 2009, A\&A, 498, 877

Carlsson, M. 1986, Uppsala Astronomical Observatory Report No. 33

Centeno, R., \& Socas-Navarro, H. 2008, ApJ, 682, L61

Chmielewski, Y., Brault, J. W., \& Mueller, E. A. 1975, A\&A, 42, 37

Christensen-Dalsgaard, J., Monteiro, M. J. P. F. G., \& Thompson, M. J. 1995, MNRAS, 276, 283

Cunha, K., Hubeny, I., \& Lanz, T. 2006, ApJ, 647, L143

Delahaye, F., \& Pinsonneault, M. 2006, ApJ, 649, 529

Drake, J., \& Testa, P. 2005, Nature, 436, 525

Drawin, H. W. 1968, Z. Phys., 211, 404

Evonak, M., \& Glatzmaier, G. 2004, Geophys. Astrophys. Fluid Dyn., 98, 241

Grevesse, N., \& Noels, A. 1993, Phys. Scr. T, 47, 133

Grevesse, N., \& Sauval, A. J. 1998, Space Sci. Rev., 85, 161

Guzik, J. A., Watson, L. S., \& Cox, A. N. 2005, ApJ, 627, 1049
Holweger, H. 2001, in AIP Conf. Proc. 598, Joint SOHO/ACE Workshop: Solar and Galactic Composition, ed. R. F. Wimmer-Schweingruber (Melville, NY: AIP), 23

Holweger, H., \& Mueller, E. A. 1974, Sol. Phys., 39, 19

Iglesias, C. A., \& Rogers, F. J. 1996, ApJ, 464, 943

Johansson, S., Litzén, U., Lundberg, H., \& Zhang, Z. 2003, ApJ, 584, L107

Kiselman, D. 1993, A\&A, 275, 269

Koesterke, L., Allende Prieto, C., \& Lambert, D. L. 2008, ApJ, 680, 764

Lodders, K. 2003, ApJ, 591, 1220

Meléndez, J. 2004, ApJ, 615, 1042

Meléndez, J., \& Asplund, M. 2008, A\&A, 490, 817

Neuforge-Verheecke, C., Guzik, J. A., Keady, J. J., Magee, N. H., Bradley, P. A., \& Noels, A. 2001, ApJ, 561, 450

Piau, L., \& Turck-Chièze, S. 2002, ApJ, 566, 419

Pinsonneault, M. 1997, ARA\&A, 35, 557

Reetz, J. 1998, PhD thesis, Ludwig-Maximilians Univ.

Rogers, T. M., \& Glatzmaier, G. A. 2005, ApJ, 620, 432

Rogers, T. M., Glatzmaier, G. A., \& Jones, C. A. 2006, ApJ, 653, 765

Schmelz, J. T., Nasraoui, K., Roames, J. K., Lippner, L. A., \& Garst, J. W. 2005, ApJ, 634, L197

Scott, P. C., Asplund, M., Grevesse, N. A., \& Sauval, J. 2006, A\&A, 456, 675

Scott, P. C., Asplund, M., Grevesse, N. A., \& Sauval, J. 2009, ApJ, 691, L117

Shchukina, N., \& Trujillo Bueno, J. 2001, ApJ, 550, 970

Socas-Navarro, H., \& Norton, A. A. 2007, ApJ, 660, L153

Steffen, M., \& Holweger, H. 2002, A\&A, 387, 258

Stein, R. F., \& Nordlund, A. 1998, ApJ, 499, 914

Storey, P. J., \& Zeippen, C. J. 2000, MNRAS, 312, 813

Stuerenburg, S., \& Holweger, H. 1990, A\&A, 237, 125

Turck-Chièze, S., Couvidat, S., Piau, L., Ferguson, J., Lambert, P., Ballot, J., García, R. A., \& Nghiem, P. 2004, Phys. Rev. Lett., 93, 211102

Wedemeyer, S. 2001, A\&A, 373, 998

Zhang, K., \& Schubert, G. 2006, Rep. Prog. Phys., 69, 1581 